\title{
ML Estimator and Hybrid Beamformer for Multipath and Interference Mitigation in GNSS Receivers
}

\author{
Gonzalo Seco-Granados, Member, IEEE, Juan A. Fernández-Rubio, Member, IEEE, and \\ Carles Fernández-Prades, Student Member, IEEE
}

\begin{abstract}
This paper addresses the estimation of the code-phase (pseudorange) and the carrier-phase of the direct signal received from a direct-sequence spread-spectrum satellite transmitter. The signal is received by an antenna array in a scenario with interference and multipath propagation. These two effects are generally the limiting error sources in most high-precision positioning applications. A new estimator of the code- and carrier-phases is derived by using a simplified signal model and the maximum likelihood (ML) principle. The simplified model consists essentially of gathering all signals, except for the direct one, in a component with unknown spatial correlation. The estimator exploits the knowledge of the direction-of-arrival of the direct signal and is much simpler than other estimators derived under more detailed signal models. Moreover, we present an iterative algorithm, that is adequate for a practical implementation and explores an interesting link between the ML estimator and a hybrid beamformer. The mean squared error and bias of the new estimator are computed for a number of scenarios and compared with those of other methods. The presented estimator and the hybrid beamforming outperform the existing techniques of comparable complexity and attains, in many situations, the Cramér-Rao lower bound of the problem at hand.
\end{abstract}

Index Terms-Adaptive arrays, adaptive estimation, array signal processing, beamforming, beam steering, bias, calibration, Code division multiaccess, Cramér-Rao bounds, delay estimation, early-late estimator, Global positioning system, GPS, GPS positioning, GPS receiver, GPS signal, Gold codes, interference suppression, iterative methods, low-complexity constraint, maximum likelihood estimation, maximum likelihood estimator, MLE, modeling, multipath channels, multipath environment, multipath propagation, pseudo random codes, Radio Navigation, radio receivers, reflected components, Satellite navigation systems, signal model, simulations, single-path environment, standard deviation, Time of arrival estimation.

\section{INTRODUCTION}

$\mathbf{T}$ HE term Global Navigation Satellite Systems (GNSS) is a generic expression referring to any system that enables the calculation of the user position based on signals transmitted by a constellation of satellites. At the present time, the Global Positioning System (GPS) is the only fully operational system. The European augmentation of GPS (EGNOS) and a

Manuscript received May 16, 2003; revised February 17, 2004. This work was supported in part by the Spanish Science and Technology Commission under project TIC2001-2356-C02-01 and by the Spanish Ministry of Education under Grant FPU AP2000-3893. The associate editor coordinating the review of this manuscript and approving it for publication was Dr. Chong-Yung Chi.

G. Seco-Granados is with the Electrical Engineering Department, European Space Agency (ESA), 2200 AG Noordwijk, The Netherlands (e-mail: gonzalo@ieee.org).

J. A. Fernández-Rubio and C. Fernández-Prades are with the Signal Theory and Communications Department, Polytechnic University of Catalonia, 08034 Barcelona, Spain (e-mail: juan@gps.tsc.upc.edu; carlos@gps.tsc.upc.edu).

Digital Object Identifier 10.1109/TSP.2004.842193 new system named GALILEO will be operational in the next few years. All GNSS share the same operating principle: The receiver position is computed based on the distances between the receiving antenna and a set of satellites, and the receiver determines these distances by measuring the propagation time of the signals transmitted by the satellites. This propagation time can be obtained from the delay (referred to as pseudorange or code-phase) of the complex envelope and from the carrier-phase [1].

The surprising evolution of GNSS applications has led to stringent requirements for GNSS receivers, particularly in regard to their accuracy. Augmentations such as differential operation help to reduce or eliminate many sources of errors (e.g. common-mode atmospheric, orbit-, and satellite-induced errors), but multipath remains the dominant error source in most high-precision applications and is the limiting factor in achieving the ultimate GNSS accuracy [2]. Due to the operating principle of the GNSS, only the direct signal [which is also called the line-of-sight signal (LOSS)] bears useful information about the distance between the receiver and the satellite. Significant research and development efforts have been devoted to the mitigation of multipath effects, and a number of techniques have been proposed so far. They may be classified according to a variety of criteria, e.g., real-time versus post-processing techniques and multiple versus single antenna techniques. GNSS are also subject to external interferers, which have to be cancelled in the receiver in order to make GNSS adequate for many safety-critical applications, such as aircraft automatic guidance and landing systems. Several methods can be used to mitigate narrowband interferences in single-antenna receivers, which are usually based on a linear interpolator-subtracter structure [3]. However, in general, single-antenna methods cannot combat wideband interferences.

Errors in the pseudorange and carrier-phase measurements produced by the multipath propagation have been studied in [4]-[7], among others. Only reflections correlated with the direct signal, which are usually referred to as coherent multipath, cause these errors; this is the type of reflections considered in this paper. Their main characteristic is that their relative delays with respect to the LOSS are on the order of or smaller than the inverse of the signal bandwidth. For instance, in a GPS receiver employing a delay locked loop (DLL), which is the synchronization method used in the vast majority of GNSS receivers, the multipath components may bias the pseudoranges in several tens or even a hundred of meters, and at the same time, they hamper the ambiguity resolution process needed for carrierphase ranging. The bias in the carrier phases may reach some 
centimeters (with the favorable assumption of perfect ambiguity resolution, which is not likely the case in a multipath scenario). The most widespread multipath-mitigation techniques are those based on modifications of the conventional DLL, which are realtime signal processing methods. Some of these techniques are the Narrow Correlator DLL [7], the Multipath Estimating DLL (MEDLL) [8], the Pulse Aperture Correlator (PAC) (patented by NovAtel Inc.), and the Edge Correlator and (Enhanced) Strobe Correlator [9], [10] (patented by Ashtech Inc.). These singlesensor techniques are able to discriminate the LOSS from the reflections only in the temporal domain, and hence, their performance is still limited for many precise applications. A number of post-processing techniques for multipath mitigation have also been proposed [6]. Since most of these techniques require data recording for several minutes or hours, they do not work in real-time and are restricted to a small number of applications.

On the other hand, spatial filtering is probably the most effective approach to combat both interference and multipath. Unlike in communication systems, the potential benefits of antenna arrays in navigation systems have not been investigated thoroughly. The use of antenna arrays in GNSS has been centered on interference mitigation; see, for example, [11]-[15]. In these works, the array processor operates directly with the received signals, and conventional array processing techniques, such as the minimum-variance or the power-inversion beamformers, are applied. Combating the multipath propagation with antenna arrays is a much more powerful but also involved approach, and it has hardly been studied in the context of GNSS. Some works in this direction are [16]-[19]. One of the major difficulties in employing an antenna array in a multipath environment is that conventional array processing techniques completely fail because of the high degree of coherence existing between the LOSS and the reflections. The maximum likelihood (ML) approach has been used for synchronization in communications (see, e.g., [20] for single-antenna and [21] and [22] for multiple-antenna systems) but never exploiting the particularities of GNSS.

The goal of this paper is to present real-time (not post- or data-processing) array processing techniques that can be implemented in a GNSS receiver in order to mitigate the effects of interferences and any kind of multipath. ${ }^{1}$ The ML estimator of the pseudorange and the carrier-phase derived from a simplified model of the received signals is proposed and analyzed. The equivalence with a hybrid beamformer is shown, which leads to an iterative algorithm.

\section{SIGNAL MODEL}

This section presents the formulation of the signals received by the antenna array and justifies the simplified model on which the rest of the paper is based.

\section{A. Description of the Received Signals}

Let us consider that an arbitrary $m$-antenna array receives the signal transmitted by a given GNSS satellite. Assume also that, besides the LOSS, $d-1$ reflections of the GNSS signal impinge

\footnotetext{
${ }^{1}$ In the GNSS literature, it is normal to differentiate between specular (a few reflections produced by smooth surfaces) and diffuse (a large number of weaker reflections) multipath.
}

on the array. Then, the complex baseband representation of the signal received at the array is the $m \times 1$ vector $^{2}$

$$
\mathbf{y}[n]=\boldsymbol{\alpha}_{0} s\left(n T_{s}-\tau_{0}\right)+\sum_{k=1}^{d-1} \boldsymbol{\alpha}_{k} s\left(n T_{s}-\tau_{k}\right)+\mathbf{z}[n]
$$

where $T_{s}$ is the sampling period, $\boldsymbol{\alpha}_{k}$ and $\tau_{k}$ are the spatial signature and the time-delay of the $k$ th component, and $\mathbf{z}[n]$ models the thermal noise and all other interference. The subscript 0 stands for the LOSS. Hence, $\tau_{0}$, when expressed in length units, is the pseudorange, and the phases of the elements of $\boldsymbol{\alpha}_{0}$ are the carrier-phase observables at each antenna. The underlying analog signal $s(t)$ represents the contribution of one the signals transmitted by a GNSS satellite; it is considered to be a DirectSequence Spread-Spectrum (DS-SS) signal since all present and planned navigation systems use this modulation format [1], [23]. Therefore, the signal $s(t)$ can be expressed as

$$
s(t)=\sum_{l=-\infty}^{\infty} d(l) c(t-l T)
$$

where $c(t)$ is the spreading waveform or pseudo-noise code possibly after some kind of preprocessing. The sequence of symbols $d(l)$ forms the navigation message of the satellite, essentially bearing ephemerides information. The symbol period $T$ comprises $P$ chips, each of duration $T_{c}$, i.e., $P=T / T_{c}$. Note that $s(t)$ is not necessarily equal to the signal transmitted by the satellite since in general, the signals can be preprocessed in the receiver before applying any array processing algorithm. In our case, the need for this preprocessing arises from the fact that the received GNSS signals are well below the noise floor. If the array processor operated directly on the received signals, it would not be possible to infer any spatial information about the reflected replicas. That is to say, the array would be insensitive to the direct and reflected signals, and it would be able to cancel only powerful interferences, as in [12]-[15], because the contribution to the spatial correlation matrix would be dominated by the noise and interference. Since our overall objective is to use the spatial dimension to cancel both multipath components and interference, some kind of preprocessing that increases the signal-to-noise ratio (SNR) of the LOSS, and its reflections, is mandatory. When dealing with DS-SS signals, the standard approach to attain this purpose is to perform the despreading (i.e., simply to correlate the received signal with a local replica of the pseudo-noise code). In any case, the advantage of the techniques proposed in the following section is that they are independent of the particular way $s(t)$ is obtained.

An assumption needed by the techniques proposed in this paper is that $s(t)$ is known. This assumption does not represent any limitation because of the following.

- $\quad$ The underlaying shaping pulse in $s(t)$ (i.e., $c(t)$ ) is known at the receiver (it is a design parameter of the system).

- The techniques can be applied to portions of $\mathbf{y}[n]$ that span one symbol interval, and in this case, the knowledge of the transmitted symbol is not needed.

\footnotetext{
${ }^{2}$ Vectors are denoted by lowercase bold letters, and matrices are denoted by uppercase bold letters.
} 
- $\quad$ Only when the observed portion of $\mathbf{y}[n]$ spans more than one symbol interval, the symbol transitions need to be known. Even this situation is not a handicap since GNSS transmits training sequences [1], which can be used, for instance, to adapt the weights of the hybrid beamformer presented in Section V. The key issue in GNSS and in this paper is accurate synchronization and not data detection, which usually is not a problem in GNSS, even in the presence of strong interference and without using training sequences, if appropriate techniques are employed (e.g., [3], [11]-[15]). This is also facilitated by the large redundancy present in the system: The same parts of the message are transmitted at different time periods and by different satellites.

The standard "narrowband assumption" used in many array signal processing problems has been made in writing (1). This is well justified because the bandwidth of the GNSS signals is on the order of few megahertz, and the carrier frequency is between 1 and $2 \mathrm{GHz}$.

\section{B. Simplified Signal Model}

A number of techniques that estimate the unknown parameters of the detailed (as opposed to simplified) signal model in (1) have been developed. Many of them assume that the spatial signatures are parameterized by the corresponding directions-of-arrival (DOA), as in [24] and [25]. While these methods may exploit the full space-time structure of the signals, they involve the optimization of multidimensional nonlinear cost functions. Only a few cases that resort to particular array configurations allow a closed-form estimation of the delays and DOAs, e.g., [25]. To obtain simpler criteria, most methods presume that the noise $\mathbf{z}[n]$ is spatially white, which makes them incapable of mitigating directional interferences. The large computational load of the previous techniques can be alleviated by using an unstructured parameterization of the spatial signatures, which are modeled as arbitrary deterministic vectors, as in [18] and [21].

The large complexity of all the methods enumerated above, which are based on the model (1), is mainly due to the fact that an important effort is devoted to estimating certain parameters that are not of interest in a GNSS receiver, such as the DOAs and/or the time-delays of the reflections. This justifies the search of a simpler technique allowing us to estimate only the relevant parameters. Such a technique should, however, be capable of using the diversity introduced by the antenna array in order to discriminate the signals in the spatial dimension. The proposed technique will be based on the use of a simplified signal model.

A particularity of the GNSS systems is that the receiver has accurate estimates of its own position as well as that of the satellite. Therefore, assuming that the antenna array is calibrated, it is possible to know the spatial signature of the LOSS up to a scaling factor, since the DOA of the direct signal can be computed beforehand. We will consider that the following relation holds:

$$
\boldsymbol{\alpha}_{0}=\alpha_{0} \mathbf{a}_{0}
$$

where $\mathbf{a}_{0}$ is the known steering vector of the LOSS, and $\alpha_{0}$ is an unknown complex amplitude. This is a rather common

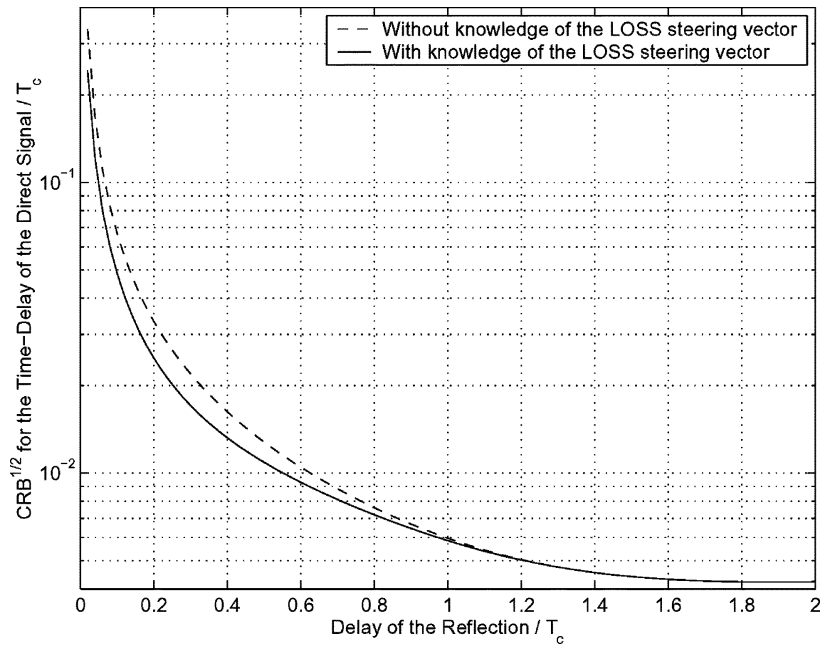

Fig. 1. CRB for the LOSS time-delay with and without a priori knowledge of $\mathbf{a}_{0}$. Parameters as in Fig. 3 except for $\check{\theta}_{1}=5^{\circ}$.

assumption in many GPS-related papers [11]-[14], [19]. The attitude of the antenna array is also needed for the computation of $\mathbf{a}_{0}$, and this information is available in many applications, as in the static receivers present in the large number of differential reference stations. In a dynamic environment, it usually requires the use of an attitude sensor or data from the navigation unit. Even in this case, in aeronautical applications, the attitude is mostly available. Furthermore, even in very adverse multipath scenarios, the inaccuracies of the satellite and receiver positions, which are at most on the order of a few hundred meters, result in negligible errors in the calculation of the DOA of the LOSS because the distance between the receiver and the satellite is larger than $20000 \mathrm{~km}$.

Another justification for the simplified signal model comes from the comparison of the two Cramér-Rao bounds (CRBs) for the LOSS time-delay estimate obtained under the detailed signal model (1) when $\mathbf{a}_{0}$ is known and when it is unknown. Fig. 1 contains one particular example of these CRBs (the scenario parameters are described in Section VI-A together with the figure caption), but the conclusions are general. Both CRBs are very close to each other and are indistinguishable in most cases. This implies that the knowledge of $\mathbf{a}_{0}$ does not provide essential information for the detailed signal model and does not allow us to improve significantly the performance of the ML estimator for that model. Moreover, that ML estimator would not result in a computational efficient implementation. Therefore, instead of using the a priori knowledge of $\mathbf{a}_{0}$ together with the detailed signal model, it is preferred to exploit this additional information in order to simplify the signal model itself, that is to say, to use a simple model (and hence simple estimators) that would be unfeasible without the knowledge of $\mathbf{a}_{0}$.

In the simplified signal model, the actual received signal (1) is expressed as the addition of only two terms

$$
\mathbf{y}[n]=\alpha_{0} s\left(n T_{s}-\tau_{0}\right)+\mathbf{e}[n]
$$

together with the assumption in (3) and $\mathbf{a}_{0}$ being known. The first term is the LOSS contribution, and the second one is an equivalent noise that includes the contribution of all the undesired signals, i.e., reflections, interferences, and thermal noise. 
This model is appropriate for our goals because only the desired parameters, that is, $\tau_{0}$ and $\boldsymbol{\alpha}_{0}$, remain explicitly. This fact provides additional support for separating the contribution of the direct signal from that of the rest of the signals.

Vector $\mathbf{e}[n]$ is modeled as a complex, circularly-symmetric, zero-mean Gaussian process that has an unknown and arbitrary spatial correlation matrix $\mathbf{Q}:^{3}$

$$
\mathrm{E}\{\mathbf{e}[n]\}=0, \quad \mathrm{E}\left\{\mathbf{e}[n] \mathbf{e}^{H}[l]\right\}=\mathbf{Q} \delta_{n, l} .
$$

For simplicity, the process is assumed to be temporally white. Matrix $\mathbf{Q}$ is intended to model the directional or spatial characteristics of both interference and multipath components. Indeed, it is the fact that the correlation matrix is unknown and has to be estimated that will make the estimator capable of using the diversity introduced by the antenna array to discriminate the signals in the spatial dimension, despite the approximate modeling of $\mathbf{e}[n]$. The simplification of the signal model comes clearly at the expense of a certain mismatch between the model and the actual received signals because the previous assumptions about the equivalent noise need not be satisfied in a real scenario. For instance, the zero-mean assumption of $\mathbf{e}[n]$ (or, equivalently, the assumption that signal and noise terms are uncorrelated) is violated when $\mathbf{e}[n]$ contains the contribution of (coherent) reflections. However, this mismatch also has some positive effects on the performance of the estimators, as further discussed in Section VI-C. The Gaussian hypothesis for the equivalent noise is of interest because it allows an analytical treatment of the ML estimator and easily captures in $\mathbf{Q}$ the spatial structure of the multipath and interference.

All in all, the use of the simplified signal model is justified by the fact that it allows derivation of simple estimators, whose performance is excellent, as shown in Section VI. Indeed, the same idea has been applied successfully to synchronization in mobile communication systems with multiuser interference (see, e.g., [22], [26], and [27]) and to Doppler and DOA estimation in radar systems (see, e.g., [28]).

The $N$ samples of (4) collected during an observation interval can be arranged into the following $m \times N$ matrix:

$$
\mathbf{Y}=[\mathbf{y}[1] \mathbf{y}[2] \cdots \mathbf{y}[N]]=\alpha_{0} \boldsymbol{a}_{0} \boldsymbol{s}^{T}\left(\tau_{0}\right)+\mathbf{E}
$$

where $\mathbf{E}$ is formed identically to $\mathbf{Y}$, and we have defined the signal vector

$$
\mathbf{s}(\tau)=\left[s\left(T_{s}-\tau\right) s\left(2 T_{s}-\tau\right) \cdots s\left(N T_{s}-\tau\right)\right]^{T} .
$$

\section{ML Code AND CARrier-Phase Estimators}

The problem addressed in this section may be stated as follows: Given the collection of data $\mathbf{Y}$ modeled by (6), the vector $\mathbf{a}_{0}$, and the signal $s(t)$, estimate the unknown parameters $\tau_{0}, \alpha_{0}$, and $\mathbf{Q}$. To this end, the ML principle is going to be applied. The inverse of the likelihood function of the data is ${ }^{4}$

$$
\Lambda_{1}\left(\tau_{0}, \alpha_{0}, \mathbf{Q}\right)=|\mathbf{Q}| \exp \left\{\operatorname{Tr}\left\{\mathbf{Q}^{-1} \mathbf{C}\left(\tau_{0}, \alpha_{0}\right)\right\}\right\}
$$

${ }^{3}$ The transpose, conjugate, and conjugate transpose operations are designated by $(\cdot)^{T},(\cdot)^{*}$, and $(\cdot)^{H}$, respectively. $\delta_{n, l}$ is equal to 1 if $n=l$ and 0 otherwise. $\mathrm{E}\{\cdot\}$ is the mathematical expectation.

${ }^{4}$ Throughout the paper, all parameter-independent additive or multiplicative constants in likelihood functions are neglected. $\operatorname{Tr}\{\cdot\}$ denotes the trace operation. $|\cdot|$ denotes the determinant for matrices and the absolute value for scalars. where

$$
\mathbf{C}\left(\tau_{0}, \alpha_{0}\right)=\frac{1}{N}\left(\mathbf{Y}-\alpha_{0} \mathbf{a}_{0} \mathbf{s}^{T}\left(\tau_{0}\right)\right)\left(\mathbf{Y}-\alpha_{0} \mathbf{a}_{0} \mathbf{s}^{T}\left(\tau_{0}\right)\right)^{H} .
$$

The ML estimates of the parameters ( $\hat{\tau}_{\mathrm{ML}}, \hat{\alpha}_{\mathrm{ML}}$, and $\left.\hat{\mathbf{Q}}_{\mathrm{ML}}\right)$ are those values that minimize (8). The value of $\mathbf{Q}$ that nulls the gradient of (8) with respect to $\mathbf{Q}$ is given by

$$
\hat{\mathbf{Q}}_{\mathrm{ML}}=\left.\mathbf{C}\left(\tau_{0}, \alpha_{0}\right)\right|_{\hat{\tau}_{0, \mathrm{ML}}, \hat{\alpha}_{0, \mathrm{ML}}},
$$

where we have assumed that $N \geq m+1$ in order for $\mathbf{C}\left(\tau_{0}, \alpha_{0}\right)$ to be invertible with probability one.

Let us define the following sample correlations:

$$
\begin{aligned}
\hat{P}_{s} & =\frac{1}{N} \mathbf{s}^{H}\left(\tau_{0}\right) \mathbf{s}\left(\tau_{0}\right), \hat{\mathbf{r}}_{y s}\left(\tau_{0}\right)=\frac{1}{N} \mathbf{Y} \mathbf{s}^{*}\left(\tau_{0}\right) \\
\hat{\mathbf{R}}_{y y} & =\frac{1}{N} \mathbf{Y} \mathbf{Y}^{H}, \hat{\mathbf{W}}\left(\tau_{0}\right)=\hat{\mathbf{R}}_{y y}-\hat{P}_{s}^{-1} \hat{\mathbf{r}}_{y s}\left(\tau_{0}\right) \hat{\mathbf{r}}_{y s}^{H}\left(\tau_{0}\right) .
\end{aligned}
$$

Matrix $\hat{\mathbf{W}}\left(\tau_{0}\right)$ is an unstructured estimate of the noise correlation matrix $\mathbf{Q}$. It is called unstructured because, unlike $\mathbf{C}\left(\tau_{0}, \alpha_{0}\right)$, it does not use the knowledge of the spatial signature of the LOSS. Substituting (10) into (8) yields the following concentrated inverse likelihood function:

$$
\begin{aligned}
& \Lambda_{2}\left(\tau_{0}, \alpha_{0}\right)=\mid \hat{\mathbf{R}}_{y y}-\alpha_{0} \mathbf{a}_{0} \hat{\mathbf{r}}_{y s}^{H}\left(\tau_{0}\right)-\alpha_{0}^{*} \hat{\mathbf{r}}_{y s}\left(\tau_{0}\right) \mathbf{a}_{0}^{H} \\
& +\left|\alpha_{0}\right|^{2} \hat{P}_{s} \mathbf{a}_{0} \mathbf{a}_{0}^{H} \mid \\
& =\mid \hat{\mathbf{W}}\left(\tau_{0}\right)+\left(\alpha_{0} \mathbf{a}_{0}-\hat{\mathbf{r}}_{y s}\left(\tau_{0}\right) \hat{P}_{s}^{-1}\right) \\
& \times \hat{P}_{s}\left(\alpha_{0} \mathbf{a}_{0}-\hat{\mathbf{r}}_{y s}\left(\tau_{0}\right) \hat{P}_{s}^{-1}\right)^{H} \\
& =\left|\hat{\mathbf{W}}\left(\tau_{0}\right)\right| \\
& \times\left(1+\hat{P}_{s}\left(\alpha_{0} \mathbf{a}_{0}-\hat{\mathbf{r}}_{y s}\left(\tau_{0}\right) \hat{P}_{s}^{-1}\right)^{H} \hat{\mathbf{W}}^{-1}\left(\tau_{0}\right)\right. \\
& \left.\times\left(\alpha_{0} \mathbf{a}_{0}-\hat{\mathbf{r}}_{y s}\left(\tau_{0}\right) \hat{P}_{s}^{-1}\right)\right) .
\end{aligned}
$$

Equation (15) stems directly from the following property of the determinant: $|\mathbf{I}+\mathbf{U V}|=|\mathbf{I}+\mathbf{V U}|$, which is valid for matrices with the appropriate dimensions. A straightforward minimization of (15) with respect to $\alpha_{0}$ results in the ML estimate of the LOSS amplitude:

$$
\hat{\alpha}_{0, \mathrm{ML}}=\left.\frac{\mathbf{a}_{0}^{H} \hat{\mathbf{W}}^{-1}\left(\tau_{0}\right) \hat{\mathbf{r}}_{y s}\left(\tau_{0}\right)}{\hat{P}_{s} \mathbf{a}_{0}^{H} \hat{\mathbf{W}}^{-1}\left(\tau_{0}\right) \mathbf{a}_{0}}\right|_{\tau_{0}=\hat{\tau}_{0, \mathrm{ML}}} .
$$

Thanks to the invariance principle of the ML estimates, the ML estimate of the carrier phase is directly the phase of $\hat{\alpha}_{0, \mathrm{ML}}$. After plain but lengthy calculations, in which (16) is substituted into (15) and $\hat{\mathbf{W}}^{-1}\left(\tau_{0}\right)$ is expanded using the matrix inversion lemma, the criterion in (15) can be expressed as a function of $\tau_{0}$ only:

$$
\begin{aligned}
\Lambda_{3}\left(\tau_{0}\right)= & \Lambda_{2}\left(\tau_{0}, \hat{\alpha}_{0, \mathrm{ML}}\right) \\
= & \left|\hat{\mathbf{R}}_{y y}\right|\left(1-\hat{\alpha}_{0, \mathrm{ML}} \hat{\mathbf{r}}_{y s}^{H} \hat{\mathbf{R}}_{y y}^{-1} \mathbf{a}_{0}\right) \\
= & \left|\hat{\mathbf{R}}_{y y}\right| \\
& \times\left(1+\frac{\left|\mathbf{a}_{0}^{H} \hat{\mathbf{R}}_{y y}^{-1} \hat{\mathbf{r}}_{y s}\left(\tau_{0}\right)\right|^{2}}{\left(\hat{P}_{s}-\hat{\mathbf{r}}_{y s}^{H}\left(\tau_{0}\right) \hat{\mathbf{R}}_{y y}^{-1} \hat{\mathbf{r}}_{y s}\left(\tau_{0}\right)\right)\left(\mathbf{a}_{0}^{H} \hat{\mathbf{R}}_{y y}^{-1} \mathbf{a}_{0}\right)}\right)
\end{aligned}
$$


Therefore, the ML estimate of the time delay is

$$
\begin{aligned}
\hat{\tau}_{0, \mathrm{ML}} & =\arg \max _{\tau_{0}} \Lambda_{\mathrm{ML}}\left(\tau_{0}\right) \\
& \triangleq \arg \max _{\tau_{0}} \frac{\left|\mathbf{a}_{0}^{H} \hat{\mathbf{R}}_{y y}^{-1} \hat{\mathbf{r}}_{y s}\left(\tau_{0}\right)\right|^{2}}{\hat{P}_{s}-\hat{\mathbf{r}}_{y s}^{H}\left(\tau_{0}\right) \hat{\mathbf{R}}_{y y}^{-1} \hat{-\mathbf{r}_{y s}}\left(\tau_{0}\right)} .
\end{aligned}
$$

The computation of the ML estimates involves only the search of the maximum of the one-dimensional function $\Lambda_{\mathrm{ML}}\left(\tau_{0}\right)$; therefore, this method is a good candidate for implementation in a real receiver. Moreover, this ML estimator is applicable in the same way in the presence of any type (specular or diffuse) of multipath, which is an important advantage with respect to the methods based on the detailed model in (1). If the term $\mathbf{e}[n]$ is temporally correlated, the presented estimator is not the ML one, but it can be applied in the same way without expecting in general significantly worse performance because the estimator maintains the ability to cancel interferences in the spatial domain. Obviously, better performance can achieved if the temporal correlation is exploited, following, for instance, the approaches in [22] or [29], but this comes at the expense of a much higher computational complexity. No general statements about the potential improvement are possible since it depends on the spectral shape of $s(t)$ and $\mathbf{e}[n]$.

The previous ML estimator will be compared in Section VI with three other methods, which are outlined below. The first one consists in assuming that the steering vector of the LOSS is arbitrary and unknown along with the model in (4), (5). The minimization of (15) with respect to $\left(\alpha_{0} \mathbf{a}_{0}\right)$ is trivial, and the ML time-delay estimate obtained using only a temporal reference is

$$
\hat{\tau}_{0, T E}=\arg \max _{\tau_{0}} \Lambda_{\mathrm{TE}}\left(\tau_{0}\right) \triangleq \arg \max _{\tau_{0}} \frac{\hat{\mathbf{r}}_{y s}^{H}\left(\tau_{0}\right) \hat{\mathbf{R}}_{y y}^{-1} \hat{\mathbf{r}}_{y s}\left(\tau_{0}\right)}{\hat{P}_{s}} .
$$

The second method relies on the same simplified model as the ML estimator proposed in this paper but with the additional assumption that the noise is spatially white. In this method, vector $\mathbf{a}_{0}$ is again considered to be known. The derivation of the ML estimates when $\mathbf{Q}$ is replaced by $\sigma^{2} \mathbf{I}$ is simple and yields

$$
\begin{aligned}
\hat{\alpha}_{0, \mathrm{WH}} & =\left.\frac{\mathbf{a}_{0}^{H} \hat{\mathbf{r}}_{y s}\left(\tau_{0}\right)}{\hat{P}_{s} \mathbf{a}_{0}^{H} \mathbf{a}_{0}}\right|_{\tau_{0}=\hat{\tau}_{0}, \mathrm{WH}} \\
\hat{\tau}_{0, \mathrm{WH}} & =\arg \max _{\tau_{0}} \Lambda_{\mathrm{WH}}\left(\tau_{0}\right) \\
& \triangleq \arg \max _{\tau_{0}} \frac{\left|\mathbf{a}_{0}^{H} \hat{\mathbf{r}}_{y s}\left(\tau_{0}\right)\right|^{2}}{\hat{P}_{s}} .
\end{aligned}
$$

The last approach involves the spatial filtering of the received signals using the classical minimum-variance or Capon beamformer (MVB):

$$
\hat{\mathbf{w}}_{\mathrm{MV}}=\frac{\hat{\mathbf{R}}_{y y}^{-1} \mathbf{a}_{0}}{\mathbf{a}_{0}^{H} \hat{\mathbf{R}}_{y y}^{-1} \mathbf{a}_{0}} .
$$

At first glance, this may seem to be a logical solution, and it has been proposed for the problem under consideration in some works, such as [12] and [14]. If the ML criterion is applied to the output signal of the beamformer $\left(\mathbf{y}_{\mathrm{MV}}^{T}=\hat{\mathbf{w}}_{\mathrm{MV}}^{H} \mathbf{Y}\right)$, the resulting estimates are

$$
\begin{aligned}
\hat{\alpha}_{0, \mathrm{MV}} & =\left.\frac{\mathbf{a}_{0}^{H} \hat{\mathbf{R}}_{y y}^{-1} \hat{\mathbf{r}}_{y s}\left(\tau_{0}\right)}{\hat{P}_{s} \mathbf{a}_{0}^{H} \hat{\mathbf{R}}_{y y}^{-1} \mathbf{a}_{0}}\right|_{\tau_{0}=\hat{\tau}_{0, \mathrm{MV}}} \\
\hat{\tau}_{0, \mathrm{MV}} & =\arg \max _{\tau_{0}} \Lambda_{\mathrm{MV}}\left(\tau_{0}\right) \\
& \triangleq \arg \max _{\tau_{0}} \frac{\left|\mathbf{a}_{0}^{H} \hat{\mathbf{R}}_{y y}^{-1} \hat{\mathbf{r}}_{y s}\left(\tau_{0}\right)\right|^{2}}{\hat{P}_{s}} .
\end{aligned}
$$

It is very interesting to observe that the ML criterion proposed in (19) can be expressed as a function of the ML criterion using only temporal information and the cost function based on the MVB:

$$
\Lambda_{\mathrm{ML}}\left(\tau_{0}\right)=\frac{\Lambda_{\mathrm{MV}}\left(\tau_{0}\right)}{1-\Lambda_{\mathrm{TE}}\left(\tau_{0}\right)} .
$$

In Sections IV-C and VI-C2, we will dwell on the implications of this expression.

\section{PERformance ANALYSIS}

A complete characterization of the performance of the proposed ML estimator can only be obtained by simulation since analytical expressions of the bias and the variance under all possible circumstances (i.e., under the validity of the detailed model (1) and under the validity of the simplified model (4)) do not yet exist. However, it is possible to carry out an analytical study of certain characteristics, and together with the simulation results, the study reveals important features of the ML estimator. First, the asymptotic behavior of the cost function is addressed; second, the CRBs for both models are presented; and last, the effect of errors in the a priori knowledge of $\mathbf{a}_{0}$ is analyzed.

\section{A. Asymptotic Properties of the ML Cost Function}

It can be shown that if the model (3)-(5) holds exactly, the previous ML estimators of $\tau_{0}(19), \alpha_{0}(16)$, and $\mathbf{Q}(10)$ are consistent. The proof follows closely the proof in [28]. Next, we analyze the asymptotic (hereinafter, in the number of samples $N$ ) expression of the cost function (19) when the actual received signal corresponds to the detailed signal model (1). We use the following notation: $\boldsymbol{\tau}=\left[\begin{array}{lll}\tau_{0} & \cdots & \tau_{d-1}\end{array}\right]^{T} ; \mathbf{C}_{s s}(\boldsymbol{\tau}, \boldsymbol{\lambda})$ is a matrix whose $k, l$ th element is $c_{s s}\left(\lambda_{l}-\tau_{k}\right)$, where $c_{s s}(\cdot)$ is the asymptotic autocorrelation of $s(t)$ and $\lambda_{l}$ is the $l$ th element of $\boldsymbol{\lambda} ; \mathbf{A}=\left[\begin{array}{lll}\boldsymbol{\alpha}_{0} & \cdots & \boldsymbol{\alpha}_{d-1}\end{array}\right] ;($.$) denotes the true value of the cor-$ responding parameter; and $\mathbf{R}_{y y}=\mathbf{A C}_{s s}(\check{\boldsymbol{\tau}}, \check{\boldsymbol{\tau}}) \mathbf{A}^{H}+\mathbf{Q}$. The asymptotic expression of cost function $\Lambda_{\mathrm{ML}}\left(\tau_{0}\right)$, shown in (19), is

$$
\frac{\left|\mathbf{a}_{0}^{H} \mathbf{R}_{y y}^{-1} \mathbf{A} \mathbf{C}_{s s}\left(\check{\boldsymbol{\tau}}, \tau_{0}\right)\right|}{c_{s s}(0)-\mathbf{C}_{s s}^{H}\left(\check{\boldsymbol{\tau}}, \tau_{0}\right) \mathbf{A}^{H} \mathbf{R}_{y y}^{-1} \mathbf{A} \mathbf{C}_{s s}\left(\check{\boldsymbol{\tau}}, \tau_{0}\right)}
$$

with probability one. This cost function is not maximum at $\tau_{0}=$ $\check{\tau}_{0}$, in general; however, further insight can be gained by assuming a high SNR, which is the usual situation after prepro- 
cessing (see Section II-A). In the first place, the denominator of (27) can be written as (see [30])

$$
\begin{aligned}
& \frac{c_{s s}(0)}{\left|\mathbf{R}_{y y}\right|}\left|\mathbf{Q}+\mathbf{A M}\left(\check{\boldsymbol{\tau}}, \tau_{0}\right) \mathbf{A}^{H}\right| \\
& \quad=\frac{c_{s s}(0)}{\left|\mathbf{R}_{y y}\right|}|\mathbf{Q}|\left|\mathbf{I}+\mathbf{A}^{H} \mathbf{Q}^{-1} \mathbf{A M}\left(\check{\boldsymbol{\tau}}, \tau_{0}\right)\right| \\
& \quad \approx \frac{c_{s s}(0)}{\left|\mathbf{R}_{y y}\right|}|\mathbf{Q}|\left|\mathbf{A}^{H} \mathbf{Q}^{-1} \mathbf{A}\right|\left|\mathbf{M}\left(\check{\tau}, \tau_{0}\right)\right|
\end{aligned}
$$

where $\mathbf{M}\left(\check{\boldsymbol{\tau}}, \tau_{0}\right)=\mathbf{C}_{s s}(\check{\boldsymbol{\tau}}, \check{\boldsymbol{\tau}})-\mathbf{C}_{s s}\left(\check{\tau}, \tau_{0}\right) c_{s s}^{-1}(0) \mathbf{C}_{s s}^{H}\left(\check{\boldsymbol{\tau}}, \tau_{0}\right)$. The approximation in (28) is based on the assumptions of high SNR and full rank of $\mathbf{A}^{H} \mathbf{Q}^{-1} \mathbf{A}$. Matrix $\mathbf{M}\left(\check{\boldsymbol{\tau}}, \tau_{0}\right)$ is the Schur complement of $c_{s s}(0)$ in

$$
\mathbf{C}_{s s}\left(\boldsymbol{\tau}_{a}, \boldsymbol{\tau}_{a}\right)=\left[\begin{array}{c|c}
\mathbf{C}_{s s}(\check{\boldsymbol{\tau}}, \check{\boldsymbol{\tau}}) & \mathbf{C}_{s s}\left(\check{\boldsymbol{\tau}}, \tau_{0}\right) \\
\hline \mathbf{C}_{s s}^{H}\left(\check{\boldsymbol{\tau}}, \tau_{0}\right) & c_{s s}(0)
\end{array}\right]
$$

where $\boldsymbol{\tau}_{a}=\left[\check{\tau}^{T} \tau_{0}\right]^{T}$. Since $c_{s s}(0)$ is strictly greater than zero, $\mathbf{M}\left(\check{\boldsymbol{\tau}}, \tau_{0}\right)$ is singular if and only if $\mathbf{C}_{s s}\left(\boldsymbol{\tau}_{a}, \boldsymbol{\tau}_{a}\right)$ is singular. At this point, we need to consider that $\boldsymbol{s}(\tau)$ fulfils the following mild nonambiguity condition. The matrix $\mathbf{C}_{s s}(\tilde{\boldsymbol{\tau}}, \tilde{\boldsymbol{\tau}})$ is nonsingular (positive definite) for any vector $\boldsymbol{\tau}_{a}$ of length $d+1$ whose elements are all distinct. Therefore, (28) is zero when $\mathbf{C}_{s s}\left(\boldsymbol{\tau}_{a}, \boldsymbol{\tau}_{a}\right)$ is singular, and this can only happen when $\tau_{0}$ coincides with any of the elements of $\check{\tau}$, since the elements of $\check{\tau}$ are all distinct.

In the second place, we analyze the numerator of (27). Applying the matrix inversion lemma to $\mathbf{R}_{y y}$ yields

$$
\begin{aligned}
\mathbf{R}_{y y}^{-1}= & \mathbf{Q}^{-1}-\mathbf{Q}^{-1} \mathbf{A}\left(\mathbf{C}_{s s}^{-1}(\check{\boldsymbol{\tau}}, \check{\boldsymbol{\tau}})+\mathbf{A}^{H} \mathbf{Q}^{-1} \mathbf{A}\right)^{-1} \\
& \times \mathbf{A}^{H} \mathbf{Q}^{-1} \\
= & \mathbf{Q}^{-1}-\mathbf{Q}^{-1} \mathbf{A}\left(\mathbf{A}^{H} \mathbf{Q}^{-1} \mathbf{A}\right)^{-1} \\
& \times\left(\mathbf{I}+\mathbf{C}_{s s}^{-1}(\check{\tau}, \check{\boldsymbol{\tau}})\left(\mathbf{A}^{H} \mathbf{Q}^{-1} \mathbf{A}\right)^{-1}\right)^{-1} \\
& \times \mathbf{A}^{H} \mathbf{Q}^{-1}
\end{aligned}
$$

Taking into account that the SNR is high, we perform the following Taylor expansion:

$$
\begin{aligned}
\left(\mathbf{I}+\mathbf{C}_{s s}^{-1}(\check{\boldsymbol{\tau}}, \check{\boldsymbol{\tau}})\left(\mathbf{A}^{H} \mathbf{Q}^{-1} \mathbf{A}\right)^{-1}\right)^{-1} & \\
& \approx \mathbf{I}-\mathbf{C}_{s s}^{-1}(\check{\boldsymbol{\tau}}, \check{\boldsymbol{\tau}})\left(\mathbf{A}^{H} \mathbf{Q}^{-1} \mathbf{A}\right)^{-1}
\end{aligned}
$$

After substituting this expression into (31), the numerator of (27) can be expressed as

$$
\frac{1}{\left|\alpha_{0}\right|^{2}}\left|\mathbf{e}_{1}^{T} \mathbf{C}_{s s}^{-1}(\check{\boldsymbol{\tau}}, \check{\boldsymbol{\tau}}) \mathbf{C}_{s s}\left(\check{\boldsymbol{\tau}}, \tau_{0}\right)\right|^{2}
$$

where $\mathbf{e}_{1}$ is a vector whose first element is 1 and zero elsewhere. The important property is that (33) is different from zero at $\tau_{0}=$ $\check{\tau}_{0}$ and zero at $\tau_{0}=\check{\tau}_{k}$ for $k=1, \ldots, d-1$.
To sum up, the limiting expressions of both the numerator and denominator of $\Lambda_{\mathrm{ML}}\left(\tau_{0}\right)$ approximated for high SNR cancel at the true delays of the reflections (i.e., at $\tau_{0}=\check{\tau}_{k}$ for $k=$ $1, \ldots, d-1)$, whereas only the denominator is zero at the true delay of the direct signal (i.e., at $\tau_{0}=\check{\tau}_{0}$ ). Therefore, the asymptotic ML cost function tends to have a peak at the true delay of the LOSS (however, this is not a formal proof of consistency), as it will be corroborated by the numerical results. This behavior of the numerator and denominator occurs thanks to the presence of the matrix $\mathbf{R}_{y y}^{-1}$ in $\Lambda_{\mathrm{ML}}\left(\tau_{0}\right)$, which appears because the correlation of the noise has been assumed to be unknown. When the noise is considered to be spatially white, the resulting cost function is $\Lambda_{\mathrm{WH}}\left(\tau_{0}\right)$. The asymptotic value of this cost function is independent of the SNR, and it does not present the interesting properties of the numerator and denominator of the $\Lambda_{\mathrm{ML}}\left(\tau_{0}\right)$.

\section{B. Cramér-Rao Bounds}

The CRB for the detailed signal model (1) (CRB-D) can be computed using the Bangs-Slepian formula. The final expression of the CRB for the delays is

$$
\begin{aligned}
\mathbf{C R B}_{\mathbf{D}}^{-1}(\boldsymbol{\tau}) \\
=2 \operatorname{Re}\left\{\left(\mathbf{D}^{*}(\boldsymbol{\tau}) \mathbf{P}_{\mathbf{S}_{\mathbf{r}}^{\mathrm{T}}\left(\boldsymbol{\tau}_{\mathbf{r}}\right)}^{\perp} \mathbf{D}^{\mathrm{T}}(\boldsymbol{\tau})\right) \odot\left(\mathbf{A}^{\mathrm{H}} \mathbf{Q}^{-1} \mathbf{A}\right)\right\} \\
-2\left(\mathbf{s}^{H}\left(\tau_{0}\right) \mathbf{P}_{\mathbf{S}_{r}^{T}\left(\boldsymbol{\tau}_{r}\right)}^{\perp} \mathbf{s}\left(\tau_{0}\right)\right)^{-1}\left(\boldsymbol{\alpha}_{0}^{H} \mathbf{Q}^{-1} \boldsymbol{\alpha}_{0}\right)^{-1} \\
\cdot \operatorname{Re}\left\{\left(\left(\mathbf{A}^{\mathrm{H}} \mathbf{Q}^{-1} \boldsymbol{\alpha}_{0}\right) \odot\left(\mathbf{D}^{*}(\boldsymbol{\tau}) \mathbf{P}_{\mathbf{S}_{\mathbf{r}}^{T}\left(\boldsymbol{\tau}_{\mathbf{r}}\right)}^{\perp} \mathbf{S}\left(\tau_{0}\right)\right)\right)\right. \\
\left.\times\left(\left(\mathbf{s}^{\mathrm{H}}\left(\tau_{0}\right) \mathbf{P}_{\mathbf{S}_{\mathbf{r}}^{\mathrm{T}}\left(\boldsymbol{\tau}_{\mathbf{r}}\right)}^{\perp} \mathbf{D}^{\mathrm{T}}(\boldsymbol{\tau})\right) \odot\left(\boldsymbol{\alpha}_{0}^{\mathrm{H}} \mathbf{Q}^{-1} \mathbf{A}\right)\right)\right\}
\end{aligned}
$$

where we have used the following definitions:

$$
\begin{aligned}
(\boldsymbol{\tau})_{r} & =\left[\begin{array}{lll}
\tau_{1} & \cdots & \tau_{d-1}
\end{array}\right]^{T}, \\
\mathbf{S}_{r}\left(\boldsymbol{\tau}_{r}\right) & =\left[\begin{array}{lll}
\mathbf{s}\left(\tau_{1}\right) & \cdots & \mathbf{s}\left(\tau_{d-1}\right)
\end{array}\right]^{T} \\
\mathbf{d}(\tau) & =\frac{d \mathbf{s}(\tau)}{d \tau}, \\
\mathbf{D}(\boldsymbol{\tau}) & =\left[\begin{array}{lll}
\mathbf{d}\left(\tau_{0}\right) & \cdots & \mathbf{d}\left(\tau_{d-1}\right)
\end{array}\right]^{T}
\end{aligned}
$$

$\mathbf{P}_{\mathbf{S}_{r}^{T}\left(\boldsymbol{\tau}_{r}\right)}^{\perp}$ is the orthogonal projector onto the orthogonal complement of the columns of $\mathbf{S}_{r}^{T}\left(\boldsymbol{\tau}_{r}\right)$, and $\odot$ represents the Schur-Hadamard (elementwise) product. Note that if $\mathbf{a}_{0}$ is unknown, the CRB reduces to the first term in (34) with $\mathbf{P}_{\mathbf{S}_{r}^{T}\left(\boldsymbol{\tau}_{r}\right)}^{\perp}$ replaced by $\mathbf{P}_{\mathbf{S}^{T}(\boldsymbol{\tau})}^{\perp}$, which is the space-time dual to the well-known CRB for the DOAs in the deterministic signal model [21]. The derivation of (34) is lengthy, and it can be found in [30] together with the expression of the CRB for the modulus and phase of $\alpha_{0}$.

When $d=1$, (34) adopts the following expression:

$$
\mathrm{CRB}^{-1}\left(\tau_{0}\right)=2\left(\boldsymbol{\alpha}_{0}^{\mathrm{H}} \mathbf{Q}^{-1} \boldsymbol{\alpha}_{0}\right)\left(\mathbf{d}^{\mathrm{H}}\left(\tau_{0}\right) \mathbf{P}_{\mathbf{s}\left(\tau_{0}\right)}^{\perp} \mathbf{d}\left(\tau_{0}\right)\right)
$$

which coincides with the CRB obtained for a signal with unknown steering vector. In the case of $d=1$, the CRB for the 
modulus and phase of the amplitude can also be easily obtained, and their expressions are 5

$$
\begin{aligned}
\mathrm{CRB}^{-1}\left(\angle \alpha_{0}\right)= & 2\left(\boldsymbol{\alpha}_{0}^{H} \mathbf{Q}^{-1} \boldsymbol{\alpha}_{0}\right)\left(\mathbf{s}^{H}\left(\tau_{0}\right) \mathbf{P}_{\mathbf{d}\left(\tau_{0}\right)}^{\perp} \mathbf{s}\left(\tau_{0}\right)\right) \\
& \times\left(1-\frac{\operatorname{Re}^{2}\left\{\mathbf{s}^{\mathrm{H}}\left(\tau_{0}\right) \mathbf{d}\left(\tau_{0}\right)\right\}}{\left\|\mathbf{s}\left(\tau_{0}\right)\right\|^{2}\left\|\mathbf{d}\left(\tau_{0}\right)\right\|^{2}}\right)^{-1} \\
\mathrm{CRB}^{-1}\left(\left|\alpha_{0}\right|\right)= & 2\left(\mathbf{a}_{0}^{H} \mathbf{Q}^{-1} \mathbf{a}_{0}\right)\left(\mathbf{s}^{H}\left(\tau_{0}\right) \mathbf{P}_{\mathbf{d}\left(\tau_{0}\right)}^{\perp} \mathbf{s}\left(\tau_{0}\right)\right) \\
& \times\left(1-\frac{\operatorname{Im}^{2}\left\{\mathbf{s}^{\mathrm{H}}\left(\tau_{0}\right) \mathbf{d}\left(\tau_{0}\right)\right\}}{\left\|\mathbf{s}\left(\tau_{0}\right)\right\|^{2}\left\|\mathbf{d}\left(\tau_{0}\right)\right\|^{2}}\right)^{-1} .
\end{aligned}
$$

As said in the previous section, if the model (3)-(5) holds, the ML estimators of $\tau_{0}, \alpha_{0}$, and $\mathbf{Q}$ are consistent, and therefore, they are also asymptotically efficient [31, Th. 6.2.3], which implies that the corresponding variances tend to (37)-(39). In the simulation results, we use the "CRB for the simplified model" (CRB-S), which is computed using (37)-(39) under the assumption that the reflections are uncorrelated with the LOSS. The CRB-S is simply computed for comparison purposes, since in the presence of correlated reflections, it is not a valid CRB as such. It is clear that the CRB-D and the CRB-S coincide in the absence of reflections.

\section{Robustness to Calibration or Pointing Errors}

The effect of calibration or pointing errors is a subject of primary interest in any method that relies on the a priori knowledge of a steering vector, such as the ML estimator in (19). We present the asymptotic variance of the estimates obtained with $\Lambda_{\mathrm{ML}}\left(\tau_{0}\right)$ and compare it to that of the estimates obtained with $\Lambda_{\mathrm{MV}}\left(\tau_{0}\right)$. We assume that the received signal satisfies the simplified model in (4) and (5), but now, $\boldsymbol{\alpha}_{0}$ is the actual spatial signature of the direct signal, whereas $\mathbf{a}_{0}$ denotes the nominal or a priori steering vector assumed by the receiver. Vector $\mathbf{a}_{0}$ is not necessarily proportional to $\boldsymbol{\alpha}_{0}$, that is to say, only in the absence of pointing or calibration errors are $\mathbf{a}_{0}$ and $\alpha_{0}$ parallel. The simulation results will show that the conclusions drawn here are also valid in the presence of reflections. The asymptotic variance for a generic $\Lambda_{t}\left(\tau_{0}\right)$ is computed as

$$
\sigma_{t}^{2}=\frac{1}{N} \frac{\lim _{N \rightarrow \infty} N \mathrm{E}\left\{\left(\Lambda_{\mathrm{t}}^{\prime}\left(\check{\tau}_{0}\right)\right)^{2}\right\}}{\left(\lim _{N \rightarrow \infty} \Lambda_{t}^{\prime \prime}\left(\check{\tau}_{0}\right)\right)^{2}}
$$

where $(\cdot)^{\prime}$ and $(\cdot)^{\prime \prime}$ denote the first and second derivatives. The result for $\Lambda_{\mathrm{MV}}\left(\tau_{0}\right)$ is

$$
\begin{aligned}
\sigma_{\mathrm{MV}}^{2}= & \frac{1}{2\left(\mathbf{d}^{H}\left(\check{\tau}_{0}\right) \mathbf{P}_{\mathbf{s}\left(\check{\tau}_{0}\right)}^{\perp} \mathbf{d}\left(\check{\tau}_{0}\right)\right)} \\
& \times\left[\frac{\mathbf{a}_{0}^{H} \mathbf{Q}^{-1} \mathbf{a}_{0}}{\left|\mathbf{a}_{0}^{H} \mathbf{Q}^{-1} \boldsymbol{\alpha}_{0}\right|^{2}}(1+\chi)^{2}-P_{s}(2+\chi)\right]
\end{aligned}
$$

where $\chi=P_{s} \boldsymbol{\alpha}_{0}^{H} \mathbf{Q}^{-1} \boldsymbol{\alpha}_{0}$ is a measure of the SNR. If $\mathbf{a}_{0}$ is parallel to $\boldsymbol{\alpha}_{0}$, then $\sigma_{\mathrm{MV}}^{2}$ coincides with the CRB. However, (41) shows that errors in $\mathbf{a}_{0}$ strongly affect $\sigma_{\mathrm{MV}}^{2}$ since $\chi$ is typically

\footnotetext{
$5 \angle$ denotes the phase of a complex number.
}

much greater than one, which is in line with the known behavior of the minimum-variance beamformer.

The computation of the asymptotic variance for $\Lambda_{\mathrm{ML}}\left(\tau_{0}\right)$ is not affordable starting from (19), but it is possible if the relation in (26) is exploited; however, the proof is lengthy and can be found in [30]. The result is

$$
\sigma_{\mathrm{ML}}^{2}=\frac{\mathbf{a}_{0}^{H} \mathbf{Q}^{-1} \mathbf{a}_{0}}{2\left(\mathbf{d}^{H}\left(\check{\tau}_{0}\right) \mathbf{P}_{\mathbf{s}\left(\check{\tau}_{0}\right)}^{\perp} \mathbf{d}\left(\check{\tau}_{0}\right)\right)\left|\mathbf{a}_{0}^{H} \mathbf{Q}^{-1} \boldsymbol{\alpha}_{0}\right|^{2}} .
$$

The variance $\sigma_{\mathrm{ML}}^{2}$ coincides with the $\mathrm{CRB}$ when $\mathbf{a}_{0}$ is parallel to $\boldsymbol{\alpha}_{0}$, as expected. The interesting result here is that $\sigma_{\mathrm{ML}}^{2}$, unlike $\sigma_{\mathrm{MV}}^{2}$, is not very sensitive to errors in $\mathbf{a}_{0}$, even though $\Lambda_{\mathrm{MV}}\left(\tau_{0}\right)$ is the numerator of $\Lambda_{\mathrm{ML}}\left(\tau_{0}\right)$. The sensitivity of $\sigma_{\mathrm{ML}}^{2}$ is independent of the SNR and is simply given by the array beam pattern in the norm of $\mathbf{Q}^{-1}$.

\section{ITERATIVE ALgORITHM Hybrid BEAMFORMING}

In this section, the equivalence between the maximum likelihood estimation and a specific type of beamforming is presented. This equivalence is the basis for an iterative realization of the ML estimator. From the beamforming viewpoint, the cost function to be optimized has a clear interpretation and can be written without the need of a probabilistic description of the data. Thus, it is easier to understand how the signals received at different antennas are combined in order to mitigate the effects of undesired components. However, no a priori claims to the optimality of the estimates obtained with the beamforming approach can be laid. On the other hand, the maximum likelihood principle provides a procedure to obtain "optimum" estimates (in the sense that they usually are asymptotically efficient) based on a stochastic model, but sometimes, it fails in giving an understandable interpretation about how the signals are processed. First, we prove the equivalence between the ML and the beamforming approaches, and next, we present the iterative algorithm.

\section{A. Equivalence Between ML Estimator and Hybrid Beamformer}

The mean squared error (MSE) between the output of a beamformer with weights $\mathbf{w}$ and the reference signal $\alpha_{0} \mathbf{S}\left(\tau_{0}\right)$ is

$$
J_{1}\left(\mathbf{w}, \alpha_{0}, \tau_{0}\right)=\frac{1}{N}\left\|\mathbf{w}^{H} \mathbf{Y}-\alpha_{0} \mathbf{s}^{T}\left(\tau_{0}\right)\right\|^{2}
$$

where $\|\cdot\|$ denotes the 2-norm of a vector. Although (43) recalls the one that is minimized in the design of a conventional temporal-reference beamformer, there is an important difference: In (43), the reference signal is not completely known, but it is parameterized by the delay of the direct signal $\left(\tau_{0}\right)$, which has to be estimated together with the optimum weight vector. It can be shown that if (43) were minimized with the constraint $\left|\alpha_{0}\right|^{2}=1 / \hat{P}_{s}$, the resulting criterion for the delay would be $\Lambda_{\mathrm{TE}}\left(\tau_{0}\right)$. However, since the steering vector of the LOSS is known, a spatial constraint on $\mathbf{w}$ can be imposed in order to force the beamformer not to cancel that signal. Thus, the optimum weight vector $\left(\hat{\mathbf{w}}_{h \mathrm{MSE}}\right)$ and the estimates of the amplitude $\left(\hat{\alpha}_{0, h \mathrm{MSE}}\right)$ and the delay $\left(\hat{\tau}_{0, h \mathrm{MSE}}\right)$ obtained with the new 
criterion are the solutions of the following constrained optimization problem:

$$
\begin{aligned}
& \hat{\mathbf{w}}_{h \mathrm{MSE}}, \hat{\alpha}_{0, h \mathrm{MSE}}, \hat{\tau}_{0, h \mathrm{MSE}} \\
& \quad=\arg \min _{\mathbf{w}, \alpha_{0}, \tau_{0}} J_{1}\left(\mathbf{w}, \alpha_{0}, \tau_{0}\right) \\
& \text { subject to } \mathbf{w}^{H} \mathbf{a}_{0}=1 .
\end{aligned}
$$

Note that due to the spatial constraint, the value of the amplitude cannot be fixed. The resulting $\hat{\mathbf{w}}_{h \mathrm{MSE}}$ is a hybrid beamformer because it is derived using both temporal and spatial references. In addition, note that no assumptions about the spatial or statistical properties of the noise have been required to phrase this problem. The amplitude that minimizes the cost function $J_{1}$ for fixed $\mathbf{w}$ and $\tau_{0}$ is $\hat{\alpha}_{0, h \mathrm{MSE}}=\mathbf{w}^{H} \hat{\mathbf{r}}_{y s}\left(\tau_{0}\right) / \hat{P}_{s}$, which substituted into (43) and using (12) results in

$J_{2}\left(\mathbf{w}, \tau_{0}\right)=\frac{1}{N}\left\|\mathbf{w}^{H}\left(\mathbf{Y}-\frac{\mathbf{r}_{y s}\left(\tau_{0}\right)}{\hat{P}_{s}} \mathbf{s}^{T}\left(\tau_{0}\right)\right)\right\|^{2}=\mathbf{w}^{H} \hat{\mathbf{W}}\left(\tau_{0}\right) \mathbf{w}$.

The weight vector for which (46) is minimized with the constraint (45), for a fixed $\tau_{0}$, is

$$
\hat{\mathbf{w}}_{h \mathrm{MSE}}=\frac{\hat{\mathbf{W}}^{-1}\left(\tau_{0}\right) \mathbf{a}_{0}}{\mathbf{a}_{0}^{H} \hat{\mathbf{W}}^{-1}\left(\tau_{0}\right) \mathbf{a}_{0}} .
$$

After replacing $\mathbf{w}$ in (46) with (47) and expanding $\hat{\mathbf{W}}^{-1}\left(\tau_{0}\right)$ with the matrix inversion lemma, we get the expression of the MSE that has to be minimized in order to obtain $\hat{\tau}_{0, h \mathrm{MSE}}$ :

$$
\begin{aligned}
J_{h \mathrm{MSE}}\left(\tau_{0}\right) & \triangleq J_{2}\left(\hat{\mathbf{w}}_{h \mathrm{MSE}}, \tau_{0}\right)=\frac{1}{\mathbf{a}_{0}^{H} \hat{\mathbf{W}}^{-1}\left(\tau_{0}\right) \mathbf{a}_{0}} \\
& =\frac{1}{\mathbf{a}_{0}^{H} \hat{\mathbf{R}}_{y y}^{-1} \mathbf{a}_{0}+\Lambda_{\mathrm{ML}}\left(\tau_{0}\right)} .
\end{aligned}
$$

This expression proves that the ML and the beamforming approaches yield identical time-delay estimates, i.e., $\hat{\tau}_{0, h \mathrm{MSE}}=$ $\hat{\tau}_{0, \mathrm{ML}}$. The proof of the equivalence concludes by noting that, after substituting (47) into $\hat{\alpha}_{0, h \mathrm{MSE}}$, the amplitude estimates are also identical, i.e., $\hat{\alpha}_{0, h \mathrm{MSE}}=\hat{\alpha}_{0, \mathrm{ML}}$.

\section{B. Iterative Algorithm Based on the Hybrid Beamformer}

In order to derive an iterative version of the ML estimator, the MSE (43) subject to the spatial constraint (45) is minimized with respect to each parameter in a different order to the one followed above. First, the optimum beamformer for given $\tau_{0}$ and $\alpha_{0}$ can be computed using the Lagrange multipliers technique, and it is denoted by $\hat{\mathbf{w}}_{h \mathrm{MSE}}^{\bullet}$ :

$$
\begin{aligned}
\hat{\mathbf{w}}_{h \mathrm{MSE}}^{\bullet}\left(\tau_{0}, \alpha_{0}\right) & =\alpha_{0}^{*} \hat{\mathbf{w}}_{\mathrm{TE}}\left(\tau_{0}\right)+\beta\left(\tau_{0}, \alpha_{0}\right) \hat{\mathbf{w}}_{\mathrm{MV}} \\
\text { where } \beta\left(\tau_{0}, \alpha_{0}\right) & =1-\alpha_{0}^{*} \mathbf{a}_{0}^{H} \hat{\mathbf{R}}_{y y}^{-1} \hat{\mathbf{r}}_{y s}\left(\tau_{0}\right) .
\end{aligned}
$$

The minimum-variance (or spatial-reference) beamformer $\hat{\mathbf{w}}_{\mathrm{MV}}$ has been defined in (23), and the temporal-reference beamformer $\hat{\mathbf{w}}_{\mathrm{TE}}$ is

$$
\hat{\mathbf{w}}_{\mathrm{TE}}\left(\tau_{0}\right)=\hat{\mathbf{R}}_{y y}^{-1} \hat{\mathbf{r}}_{y s}\left(\tau_{0}\right) .
$$

The hybrid beamformer $\hat{\mathbf{w}}_{h \mathrm{MSE}}^{\bullet}$ is a weighted linear combination of the minimum MSE beamformer calculated with only the temporal reference $\left(\hat{\mathbf{w}}_{\mathrm{TE}}\right)$ and the minimum-variance beamformer calculated with only the spatial reference $\left(\hat{\mathbf{w}}_{\mathrm{MV}}\right)$. While the hybrid beamformer tries to attenuate the reflections, the MV beamformer combines destructively the reflections with the direct signal with the aim of minimizing the total power. Conversely, the temporal-reference beamformer tends to combine constructively the reflections with the direct signal in order to increase the total signal-to-noise-plus-interference ratio (SNIR). The behavior of the latter two beamformers is not desired in a GNSS receiver. Note that if coefficient $\beta\left(\tau_{0}, \alpha_{0}\right)$ is evaluated at the ML estimate of the amplitude, then it is proportional to the inverse ML function $\Lambda_{3}\left(\tau_{0}\right)$ in (17). Second, the time-delay and amplitude estimates are the values that minimize $J_{1}\left(\hat{\mathbf{w}}_{h \mathrm{MSE}}^{\bullet}, \alpha_{0}, \tau_{0}\right)$. Considering that the hybrid beamformer is fixed, these estimates are

$$
\begin{aligned}
\hat{\tau}_{0, h \mathrm{MSE}}^{\bullet} & =\arg \max _{\tau_{0}} \frac{\left|\mathbf{y}_{h}^{T} \mathbf{s}^{*}\left(\tau_{0}\right)\right|^{2}}{\hat{P}_{s}} \\
\hat{\alpha}_{0, h \mathrm{MSE}}^{\bullet} & =\left.\frac{\mathbf{y}_{h}^{T} \mathbf{s}^{*}\left(\tau_{0}\right)}{\hat{P}_{s}}\right|_{\tau_{0}=\hat{\tau}_{0, h \mathrm{MSE}}}{ }^{\bullet}
\end{aligned}
$$

where $\mathbf{y}_{h}^{T}=\hat{\mathbf{w}}_{h \mathrm{MSE}}^{\bullet H} \mathbf{Y}$ is the beamformer output. Estimating $\tau_{0}$ and $\alpha_{0}$ from the signal $\mathbf{y}_{h}$ is actually the process realized by any GNSS receiver since the beamformer output can be considered to be the signal received by a single equivalent antenna. Consequently, the estimators in (52) and (53) could be replaced by any of the single-sensor methods implemented in conventional receivers, such as the DLL and its variants [7], [9], [32] or the MEDLL [8]. In particular, the PAC or the Enhanced Strobe Correlator (ESC) [10] can be used at the output of the hybrid beamformer as they are complementary to the spatial filtering performed by the array. Given that the hybrid beamformer attenuates the reflections, the performance of the PAC or the ESC can only improve compared to the situation in which they are employed in a single-antenna receiver. Finally, at this point, the formulation of an iterative algorithm for the computation of the hybrid beamformer and the time-delay and amplitude estimates is immediate, and it is summarized by the following steps.

1) Choose an initial value of the beamformer $\mathbf{w}^{0)}$. With the available information, the delay-and-sum beamformer is a convenient choice: $\mathbf{w}^{0)}=\mathbf{a}_{0} /\left(\mathbf{a}_{0}^{H} \mathbf{a}_{0}\right)$.

2) For $k=1,2,3, \ldots$

a) Determine the new estimates $\hat{\tau}_{0}^{k)}$ and $\hat{\alpha}_{0}^{k)}$ from the output of the beamformer $\mathbf{w}^{k-1)}$. These estimates can be obtained using (52) and (53) or any other algorithm.

b) Update the beamformer using (49), that is, $\mathbf{w}^{k)}=\hat{\mathbf{w}}_{h \mathrm{MSE}}^{\bullet}\left(\hat{\tau}_{0}^{k)}, \hat{\alpha}_{0}^{k)}\right)$. 
The convergence of this algorithm [using (52) and (53) to estimate delay and the amplitude] to the ML solution has only been proved with extensive simulations, but an analytical proof (if possible) is not available. The calculation of the weight vector in (49) could also be done recursively employing the LMS or RLS algorithms. All in all, the iterative algorithm presented here admits of a large number of further practical refinements, and it is suitable for implementation in a real receiver and real-time operation. Step $2 \mathrm{a}$ is already implemented in any GNSS receiver, and the computational load involved by Step $2 b$ is affordable with nowadays microprocessors. Moreover, it is not necessary to update the beamformer very often-only when the scenario changes; the convergence speed at initialization is not an issue here because the required initial iterations can be performed with a fix batch of data (i.e., the rate at which the iterations are performed can be much higher than the input rate of the signal samples).

\section{Simulation Results}

The ML estimator and the hybrid beamformer are analyzed and compared with other techniques in two different situations. In one of them, the effect of wideband interference is considered, whereas in the other one, the effect of a reflected ray is addressed. For the sake of completeness, results about the robustness against errors in $\mathbf{a}_{0}$ are also included. The LOSS and temporally and spatially white noise are present in all scenarios.

\section{A. Simulation Parameters}

Signal $\mathbf{s}\left(\tau_{0}\right)$ is the concatenation truncated and sampled Nyquist square-root raised cosine pulses ${ }^{6}$ [see (2) and (7)]. Each pulse $c(t)$ has one-side bandwidth equal to $(1+\alpha) /\left(2 T_{c}\right)$, and it is truncated to the interval $\left[-3 T_{c}, 3 T_{c}\right]$. The sampling period is $T_{s}=T_{c} / 2$, thus having 13 samples in each pulse. The roll-off factor $\alpha$ is 0.2 . Each pulse is often called a finger and represents a portion of the result of filtering a DS-SS signal with a code-matched filter whose length is one symbol period $T$. Note that the matched filter does not include the chip-shaping waveform but only the effect of the pseudo-random code (i.e., the sequence of chips). Statistical significance has been obtained through 500 Monte Carlo runs. The default simulation conditions are as follows.

- The observation interval comprises $K=3$ pulses, that is to say, $N=13 K=39$ samples.

- Uniform linear array (only for simplicity), with $m=6$ antennas spaced half wavelength apart.

- DOA of the LOSS: $\check{\theta}_{0}=0^{\circ}$ relative to the array broadside. Delay of LOSS: $\check{\tau}_{0}=0$.

- $\quad$ The SNR for the LOSS averaged over all the observation window is $15.87 \mathrm{~dB}$, which is equivalent to $24 \mathrm{~dB}$ when it is averaged only over the $T_{c}$-length intervals centered on the maximum of each Nyquist pulse. This value of SNR is obtained with usual system parameters, such as a Carrier-to-Noise $(\mathrm{C} / \mathrm{No})$ spectral density of $44 \mathrm{~dB}-\mathrm{Hz}$, and a chip rate and spreading factor equal

\footnotetext{
${ }^{6}$ All conclusions drawn from the simulation results are also valid for other pulse types.
}

to those of the GPS system: $1 / T_{c}=1.023 \mathrm{Mchips} / \mathrm{s}$, $P=20460$ chips/bit.

- The interference, if present, arrives from DOA $\check{\theta}_{i}=-30^{\circ}$. The signal-to-interference ratio (SIR) of the LOSS averaged over the whole observation window is $-3.13 \mathrm{~dB}$, which means that the interference is $19 \mathrm{~dB}$ above the noise level.

- The specular reflection, if present, arrives from DOA $\check{\theta}_{1}=10^{\circ}$, it is attenuated $-3 \mathrm{~dB}$ with respect to the LOSS, and both signals are in phase at the first antenna. Although the numerical results are presented for this particular value of the relative phase of the reflection, the comparisons and the conclusions derived from them are general.

\section{B. Interference Effects}

The scenario considered here coincides with the one just described in Section VI-A, assuming that no reflections are present. In this situation, all methods are unbiased, i.e., their biases are negligible compared with their standard deviations (STDs); hence, the root mean squared error (RMSE) coincides with the STDs for all practical purposes. The RMSEs of different estimators as a function of the number of pulses $K$ are plotted in Fig. 2(a) and (b). In particular, we consider the ML estimator (MLE) in (16) and (19), the estimator based on the MV beamformer (MVBE) in (24) and (25), and the ML estimator derived under the white-noise hypothesis (ML-WHE) in (21) and (22). It is observed that only the MLE attains the CRB for the time-delay, and this is achieved for a small (five or more) number of pulses, whereas there is a slight deterioration for shorter signals. The MVBE performs as well as the MLE with regard to the carrier-phase estimation, and both easily attain the CRB. This good performance of the carrier-phase MVBE can be explained as follows. First, it can be shown that (16) and (24) satisfy $\angle \hat{\alpha}_{0, \mathrm{ML}}=\angle \hat{\alpha}_{0, \mathrm{MV}}$ if they are evaluated at the same value of $\tau_{0}$. Second, $\angle \hat{\alpha}_{0, \mathrm{ML}}$ and $\angle \hat{\alpha}_{0, \mathrm{MV}}$ are quite insensitive to variations of trial delay $\tau_{0}$ [later on, an example of this behavior will be observed in Fig. 6(b)]. Therefore, the large errors in $\hat{\tau}_{0, \mathrm{MV}}$ (compared to the errors in $\hat{\tau}_{0, \mathrm{ML}}$ ) hardly affect $\angle \hat{\alpha}_{0, \mathrm{MV}}$.

The time-delay RMSE of the MVBE tends to the CRB (see Section IV-C), but it has very unsatisfactory finite-sample behavior and needs too large of a number of samples to reach the CRB. This is in line with the results reported in [33] and corroborated by Fig. 2(c), which shows that the MVB attenuates the interference much less than the temporal-reference (TRB) and hybrid (HB) beamformers. On the other hand, the MLE does not inherit those adverse finite-sample characteristics of the MVBE, despite being related to each other according to (26). As expected, the ML-WHE is severely impaired by the interference.

\section{Multipath Effects}

In the second set of simulations, a specular reflection is received, and hence, all analyzed estimators are in general biased. Note that if the scenario parameters are time invariant, then the bias will be time invariant as well; this is independent of the assumption of $\mathbf{e}[n]$ being temporally white. 


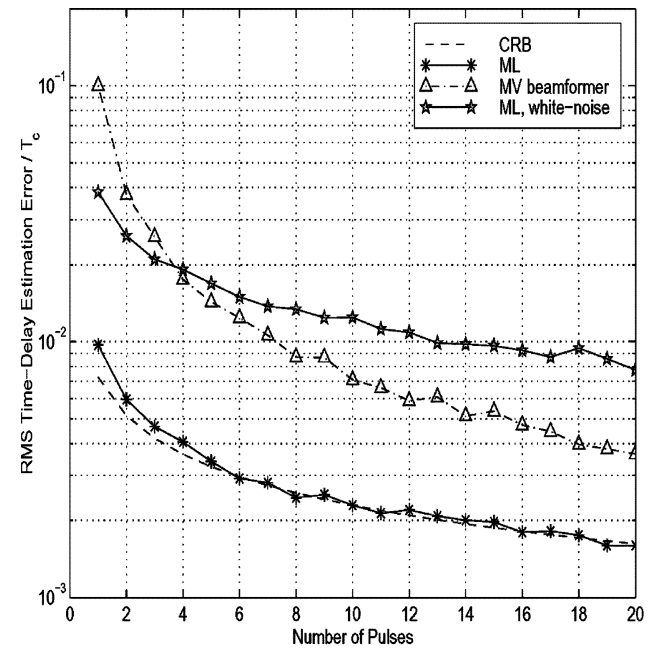

(a) Time-Delay RMSE.

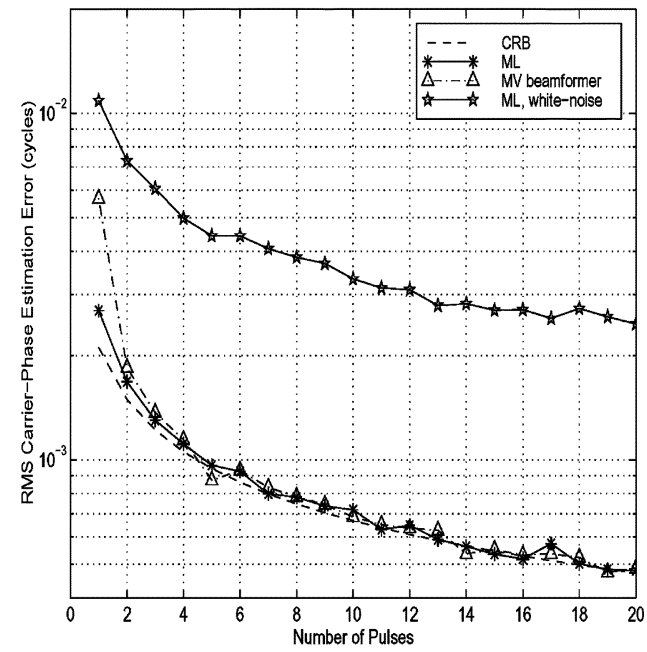

(b) Carrier-Phase RMSE.

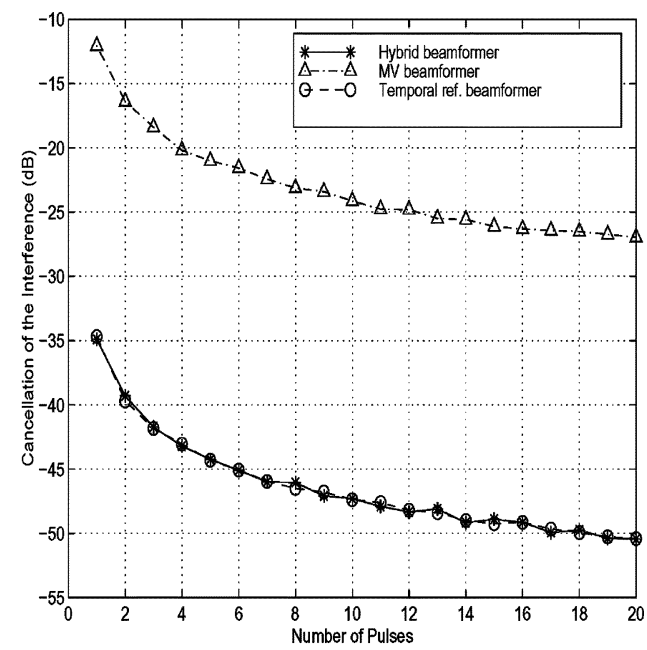

(c) Interference cancellation.

Fig. 2. Effect of varying the signals observation interval. Parameters: $\check{\theta}_{0}=0^{\circ}$, $\check{\tau}_{0}=0, \check{\theta}_{i}=-30^{\circ}, m=6$ antennas, SNR $=15.87 \mathrm{~dB}, \mathrm{SIR}=-3.13 \mathrm{~dB}$.

1) Effect of Varying the Reflection Delay: In order to assess the multipath mitigation capability of our estimator, the bias produced by a single reflection is analyzed as a function of its relative delay. This procedure is widely used in GPS literature.

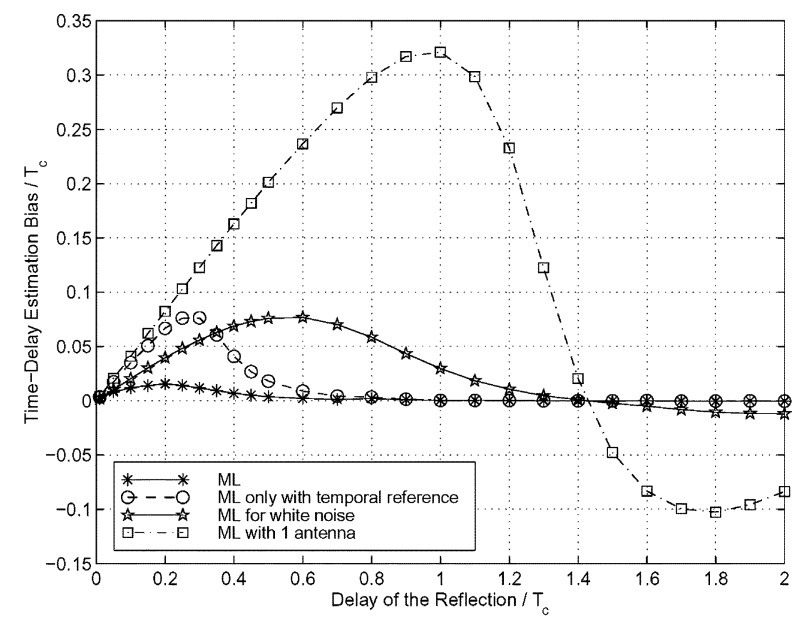

(a) Time-Delay Bias.

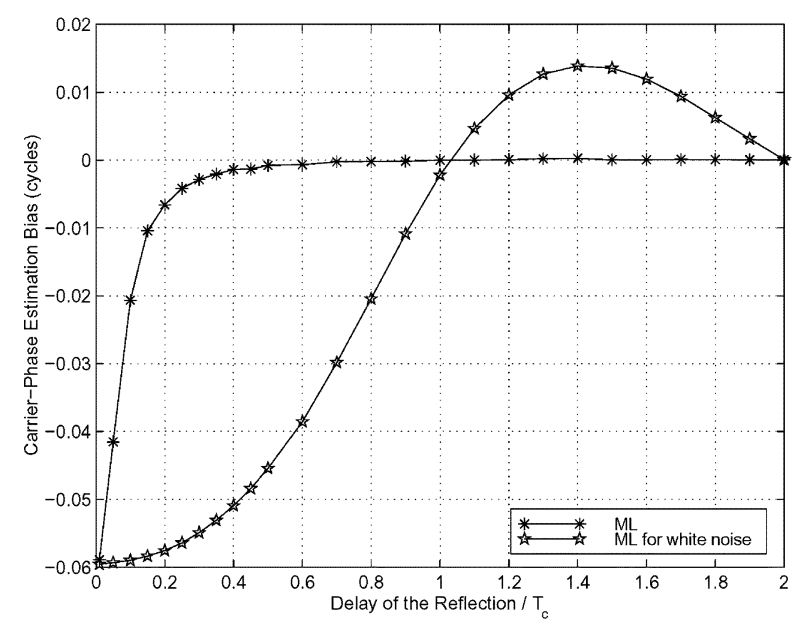

(b) Carrier-Phase Bias.

Fig. 3. Bias due to one reflection as a function of its relative delay with respect to the direct signal. Parameters: $\check{\theta}_{0}=0^{\circ}, \check{\tau}_{0}=0, \check{\theta}_{1}=10^{\circ}, \check{\alpha}_{0} / \check{\alpha}_{1}=\sqrt{2}$, $K=3$ pulses, $m=6$ antennas, $\mathrm{SNR}_{0}=15.87 \mathrm{~dB}$.

Fig. 3 shows the biases in the time-delay and carrier-phase estimates. The scenario considered here coincides with the description in Section VI-A, taking into account that no interference is received and that $\check{\tau}_{1}$ is varied. In Fig. 3(a), we have also included the bias obtained with the ML estimator when only one antenna is used; a very significant improvement is obtained by using an antenna array. As expected, the ML-WHE provides severely biased estimates because it reduces to a single-sensor ML estimator in which the reflection has been attenuated $-4.2 \mathrm{~dB}$ using the delay-and-sum beamformer. This fact also explains the fluctuations of the curves for the ML-WHE. The time-delay bias is affected by the shape of the derivative of the autocorrelation of the chip-shaping pulse $c(t)$ (which is zero around $1.42 T_{c}$ ) because the ML-WHE time-delay estimate is the value that nulls the derivative of the cross-correlation between the received signal and the reference signal [see (22)]. On the other hand, the carrier-phase bias is affected by the shape of the autocorrelation of the chip-shaping pulse $c(t)$ (which is zero at $T_{c}$ ) because the ML-WHE carrier-phase estimate is the phase of the aforementioned cross-correlation at its maximum [see (21)]. Since $s(t)$ is severely bandlimited (i.e., bandwidth $\approx 1 / T_{c}$ ), the bias obtained with the PAC or the ESC in a single-antenna 


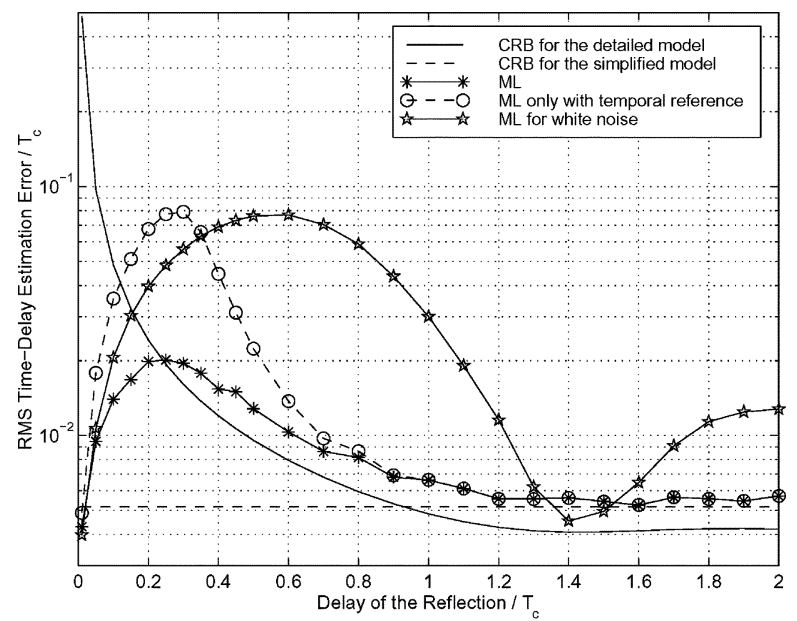

(a) Time-Delay RMSE.

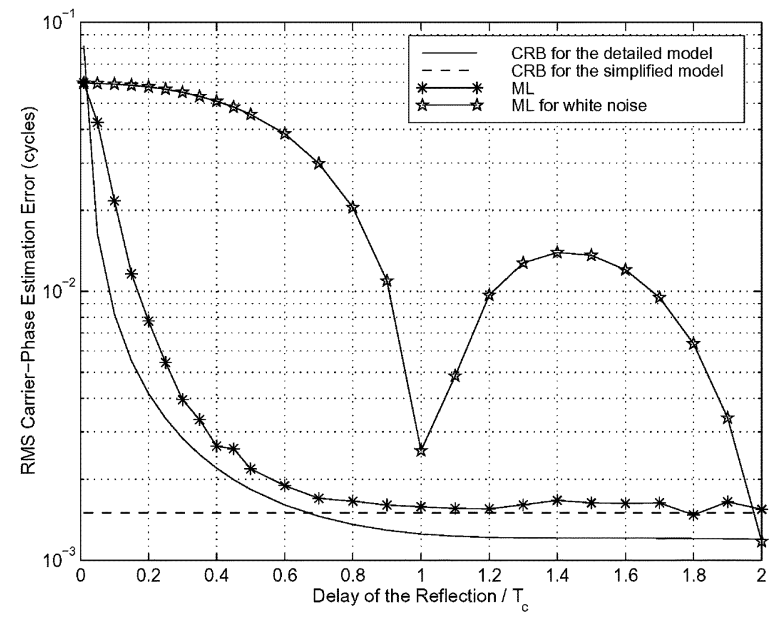

(b) Carrier-Phase RMSE.

Fig. 4. RMSE as a function of the relative delay of the reflection with respect to the direct signal. Parameters as in Fig. 3.

receiver would be close to the one shown in Fig. 3(a) for the ML estimator with one antenna [2]. Although the errors of the PAC and the ESC reported in [10] are roughly in the range of $\left[0,0.02 T_{c}\right]$ for reflection delays in the range $\left[0,0.1 T_{c}\right]$, there is no contradiction with the results in this paper because the PAC and the ESC provide such a good performance only for signals with large excess bandwidth (i.e., bandwidth $\gg 1 / T_{c}$ ). The range of delays of the reflection that cause a noticeable time-delay bias can be shortened by employing the ML estimator with only temporal reference (ML-TEE) in (20). However, a drastic reduction of the magnitude of the bias is only achieved with the MLE proposed in this paper. It has also been shown that the bias of the MLE is quite insensitive to the power of the reflection. Its performance regarding the carrier-phase bias is also excellent, even though the LOSS and the reflection arrive from close directions-of-arrival. Thanks to the use of temporal and spatial information, the MLE is able to model part of the contribution of the reflection with the unknown noise correlation matrix. This part of the reflection is considered to be an uncorrelated interference, and therefore, it is attenuated.

Next, the RMSEs as a function of the reflection delay are represented in Fig. 4. The fluctuations of the curves for the

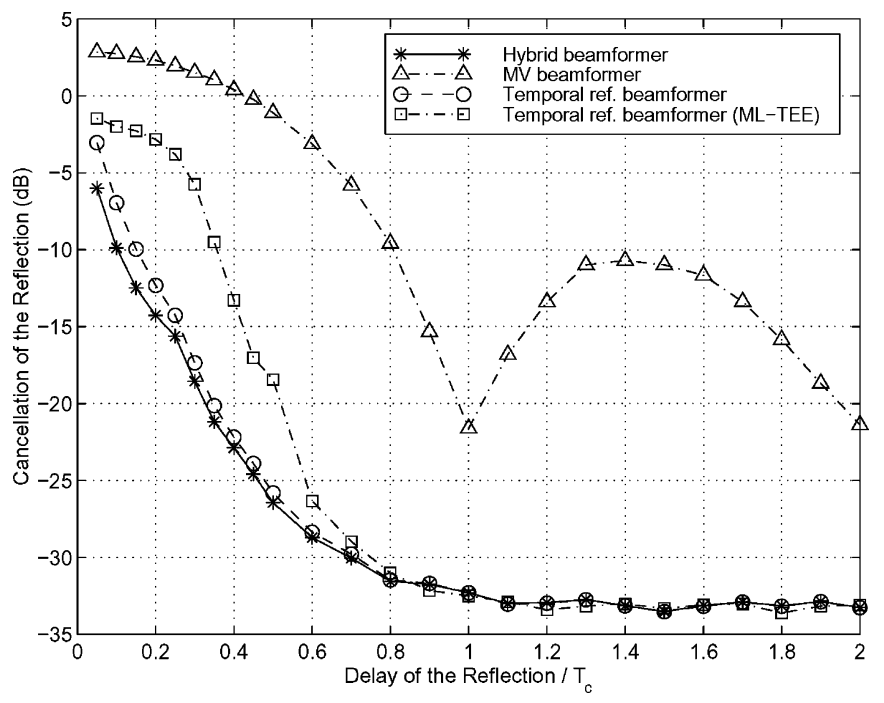

Fig. 5. Reflection cancellation with different beamformers. Parameters as in Fig. 3 and 100 iterations for the hybrid beamformer.

ML-WHE are caused by the same effect as in the case of the biases. Although the estimators are biased, the comparison with the CRB-D is still meaningful because it represents the best performance that can be achieved with more complex estimators based on the detailed model. Note that for delays $\check{\tau}_{1}>T_{c}$, it is satisfied that CRB-D $<$ CRB-S. This inequality means that it is possible to obtain a slight performance improvement by exploiting the temporal structure of the reflection, given that the LOSS and the reflection arrive from very close DOAs. If the angular separation is larger, e.g., $\left|\breve{\theta}_{1}-\breve{\theta}_{0}\right|>15^{\circ}$, the two CRBs practically coincide, which means that the temporal structure of the reflection need not be exploited when $\check{\tau}_{1}>T_{c}$. The RMSE of the MLE is smaller than that of the other methods and tends to the CRB-S for large delays, which is meaningful since the MLE does not profit from the temporal structure of the reflection. On the other hand, the RMSE of the MLE nearly reaches the CRB-D if $\check{\tau}_{1}<T_{c}$ with regard to the time-delay, or $\check{\tau}_{1}<0.7 T_{c}$, with regard to the carrier-phase, which is a surprising result. However, the RMSE does not tend to infinity when the delay spacing tends to zero, as it happens with the CRB-D. This is a remarkable advantage of the MLE proposed herein with respect to more complex unbiased estimators. By allowing a small bias, the RMSE can be smaller than the CRB-D for very closely-spaced reflections, as it is especially visible in Fig. 4(a). As a result, although the simplified model is only approximate when reflections are received, as stated in Section II-B, it makes possible the derivation of an ML estimator whose performance under certain conditions of practical interest is very close to that of other more complex methods that estimate all parameters of the reflections. Besides, this MLE offers a good tradeoff between bias and RMSE for highly coherent reflections.

The failure of the MVBE in scenarios with multipath can be explained in view of Fig. 5, which shows the cancellation of the reflection achieved with different beamformers (the hybrid beamformer used in the results in the one obtained after 100 iterations). For small delays, the MVB amplifies the reflection. The MVB offers good attenuation only when the reflection delay is 


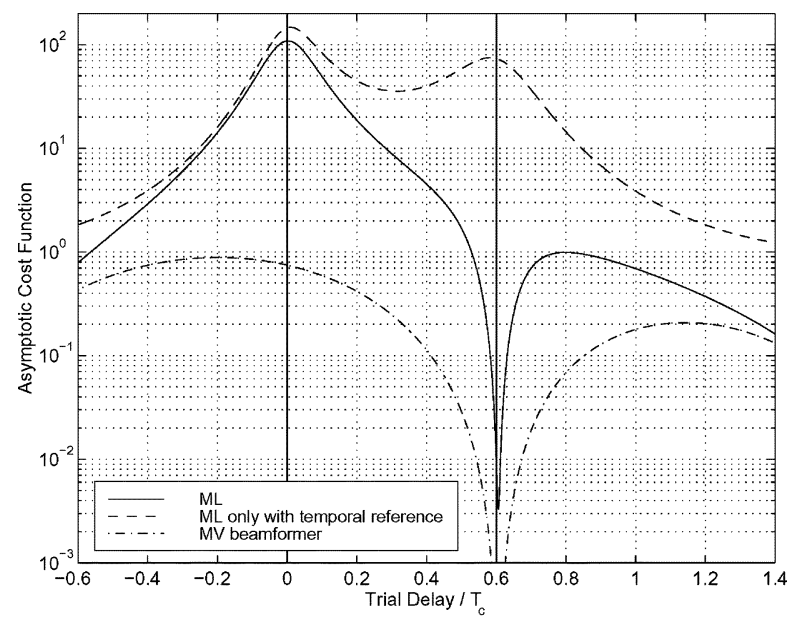

(a) Asymptotic cost functions.

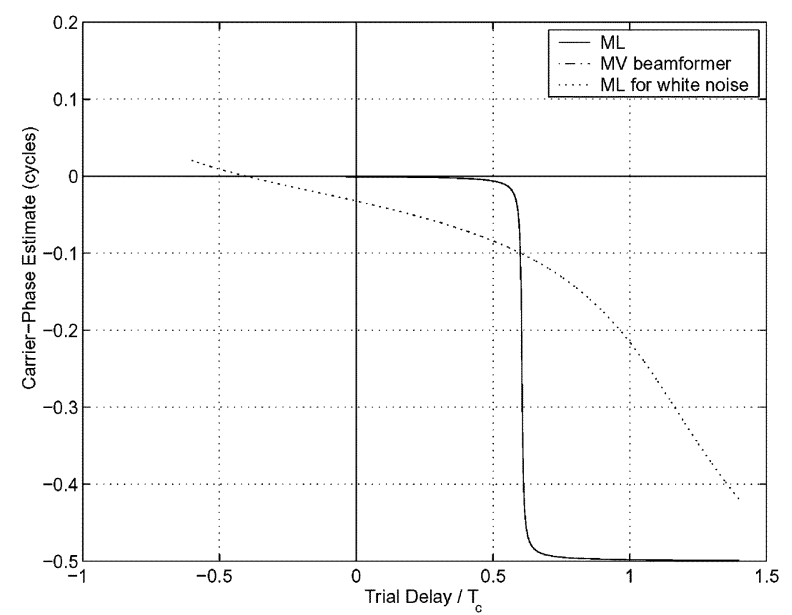

(b) Asymptotic carrier-phase estimates.

Fig. 6. Parameters: $\check{\theta}_{0}=0^{\circ}, \check{\tau}_{0}=0, \check{\theta}_{1}=10^{\circ}, \check{\tau}_{1}=0.6 T_{c}, \check{\alpha}_{0} / \check{\alpha}_{1}=$ $\sqrt{2}, K=\infty$ pulses (i.e., perfectly averaged correlations), $m=6$ antennas, $\mathrm{SNR}_{0}=15.87 \mathrm{~dB}$. Note that in (b), the lines for the ML and MVBE coincide.

a multiple of $T_{c}$, because it is only for these delays that the reflection and the LOSS are uncorrelated. The MVB response is highly dependent on the correlation of the received signals [33], and this is why its response resembles the autocorrelation of the chip-shaping pulse. The hybrid beamformer presents the highest cancellation, which increases with the separation between the LOSS and the reflection. It is interesting to note that the HB attains an attenuation of $-15 \mathrm{~dB}$ when the reflection delay $\check{\tau}_{1}$ is only $0.2 T_{c}$. On the other hand, the TRB computed with the ML-TEE estimates performs slightly worse than the TRB in the iterative process. The explanation is that the latter can be thought as being computed with the delay estimates provided by the MLE (since the HB estimates converge to the MLE ones as it will be corroborated by the results in Section VI-C2), and these estimates outperform those provided by the ML-TEE, as shown in Figs. 3 and 4.

2) Cost Functions, Effect of the SNR, and Hybrid Beamformer: In order gain further insight into why the MLE (or the HB) is preferred to both the ML-TEE (or the TRB) and the MVBE, the shape of the cost functions $\Lambda_{\mathrm{ML}}\left(\tau_{0}\right)$, $\left(1-\Lambda_{\mathrm{TE}}\left(\tau_{0}\right)\right)^{-1}$, and $\Lambda_{\mathrm{MV}}\left(\tau_{0}\right)$ is compared in Fig. 6(a). Fig. 6(b) shows the value of the carrier-phase provided by the estimators in (16), (21), and (24). In order to facilitate the comparison, we assume $\check{\tau}_{1}=0.6 T_{c}$ and $K \rightarrow \infty$ so that the effect of noise is perfectly averaged out; the rest of parameters are as in Section VI-A. According to Fig. 6(a), the ML-TEE is capable of discriminating the LOSS and the reflection, since it presents a peak for each signal. On the other hand, the MVBE is nearly flat in the vicinity of $\check{\tau}_{0}$ and presents a deep null at $\check{\tau}_{1}$. The combination of the MVBE and the ML-TEE as stated in (26) explains the good multipath mitigation capability of the MLE because, according to that equation, the MVBE selects the peak of the ML-TEE associated with the LOSS and places a null at the peak associated with the reflection. Thus, the MLE presents a single peak located near the delay of the direct signal. Note that the position of the maximum of the MLE is mainly given by the position of the corresponding peak of the ML-TEE. Nonetheless, the maximum of the MLE is slightly closer to $\check{\tau}_{0}$ than that of the ML-TEE. At the same time, the maximum of MVBE is far from $\check{\tau}_{0}$, which results in the unsatisfactory performance of the MVBE, as further commented on below. This large error of the MVBE, however, does not affect the MLE, since according to (26) and the discussion in Section IV-A, the role of the $\Lambda_{\mathrm{MV}}\left(\tau_{0}\right)$ is simply to identify which peak of $\left(1-\Lambda_{\mathrm{TE}}\left(\tau_{0}\right)\right)^{-1}$ corresponds to the LOSS.

The performance of the estimators as a function of the SNR is illustrated in Fig. 7. Although the figure shows only the results for the time-delay estimates, similar results are obtained for the carrier-phase. The signal parameters coincide with those described in Section VI-A, no interference is present, $\check{\tau}_{1}=0.25 T_{c}$, and the noise power is varied according to the SNR. When the SNR becomes very small, all the algorithms tend to have the same performance because the reflection turns out to be irrelevant with respect to the contribution of the noise, and hence, the use of the ML-WHE becomes the best solution. This also explains why the RMSE of all the estimators approaches the CRB-S for very low SNR. The bias of the ML-WHE is constant, which results in an irreducible floor level in the RMSE for high SNRs. It is corroborated that the performance of the MVBE is absolutely unsatisfactory in virtually the entire range of the $\mathrm{SNR}$, since the time-delay bias is about $-0.45 T_{c}$ for moderate or high SNR. An important characteristic observed in these figures is that the MLE and the ML-TEE are asymptotically in the SNR unbiased, as outlined in Section IV-A. Note that the RMSE of the MLE (also that of the ML-TEE) tends to the CRB-D as the SNR increases. This is a remarkable and quite surprising result, because the MLE is derived starting from the simplified model of the signals, and hence, it does not estimate the parameters of the reflections. The RMSE of the MLE is smaller than that of the ML-TEE for any SNR, mainly thanks to its reduced bias. The bias of the MLE starts decreasing at SNR $=0 \mathrm{~dB}$, which contrasts with the $20 \mathrm{~dB}$ required by the ML-TEE.

The convergence of the estimates obtained with the iterative hybrid-beamforming algorithm in Section $\mathrm{V}$ is investigated in Fig. 8(a) for two different delays of the reflection: $\check{\tau}_{1}=0.4 T_{c}$ and $\check{\tau}_{1}=T_{c}$. Although it is not shown in the figures, the carrier phase presents the same trend as the time delay, but it converges three times faster. Moreover, Fig. 8(b) and (c) illustrates the evolution of the reflection cancellation achieved by the HB and its two constituting beamformers, together with the evolu- 


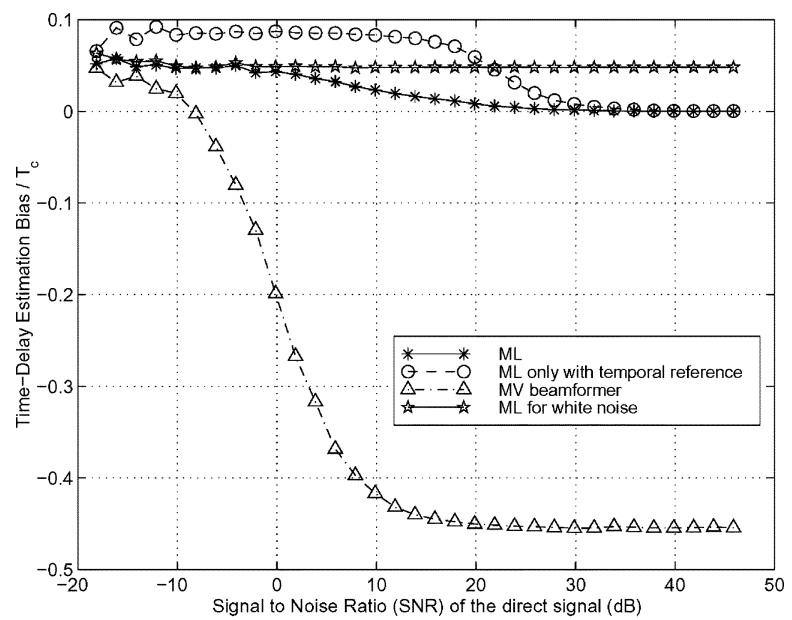

(a) Time-Delay Bias.

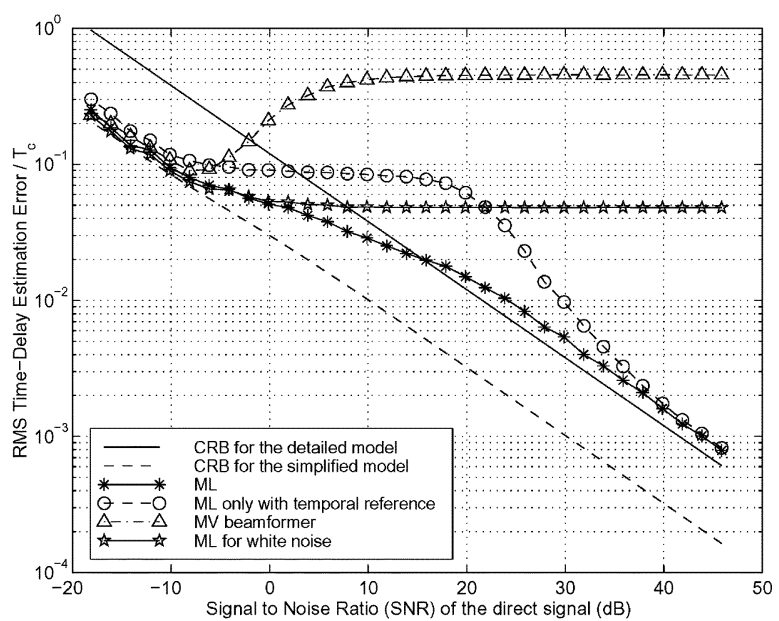

(b) Time-Delay RMSE.

Fig. 7. Performance of the proposed ML time-delay estimator as a function of the SNR (the SNR is measured as an average over the complete observation window) in the presence of one reflection. Parameters: $\check{\theta}_{0}=0^{\circ}, \check{\tau}_{0}=0, \check{\theta}_{1}=$ $10^{\circ}, \check{\tau}_{1}=0.25 T_{c}, \check{\alpha}_{0} / \check{\alpha}_{1}=\sqrt{2}, K=3$ pulses, $m=6$ antennas.

tion of the linear combination coefficients. Fig. 8(a) shows that the STD and the RMSE of the estimates obtained with the iterative algorithm tend to those of the MLE. As a matter of fact, not only is this property valid for the STD and the RMSE, but in addition, at every realization, the estimates provided by the iterative algorithm tend to the ML estimates. Despite the similar reflection cancellation offered by the $\mathrm{HB}$ and the TRB, as shown in Fig. 8(b), the knowledge of $\mathbf{a}_{0}$ is essential since it makes the iterative computation of the hybrid beamformer converge to the extraction of the parameters of the LOSS (and not those of the reflection, as it could happen if the TRB was used in the iterations).

\section{Robustness to Errors in the LOSS Steering Vector}

The effect of errors in $\mathbf{a}_{0}$ is analyzed in Fig. 9. The type of error considered is a pointing error, which is defined as the difference between the nominal DOA and the true DOA of the direct signal, namely, $\theta_{0}-\check{\theta}_{0}$. In Fig. 9(a), the reception of an interference is considered following the default parameters described in Section VI-A. It can be observed that the simulated RMSEs

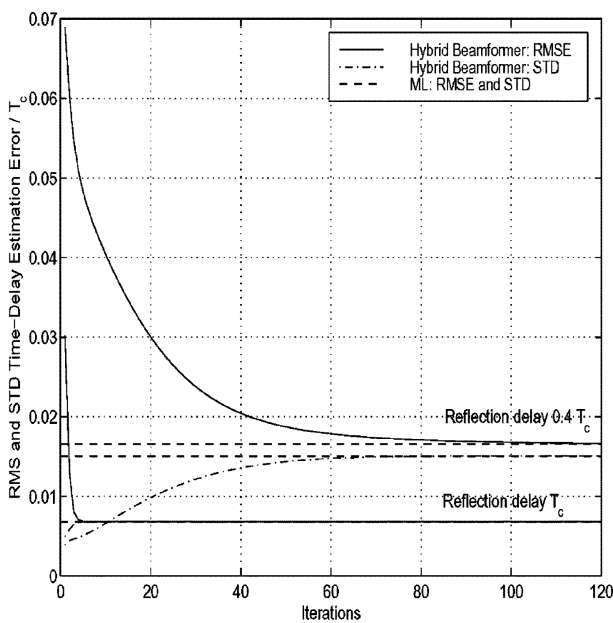

(a) Time-Delay RMSE and STD.

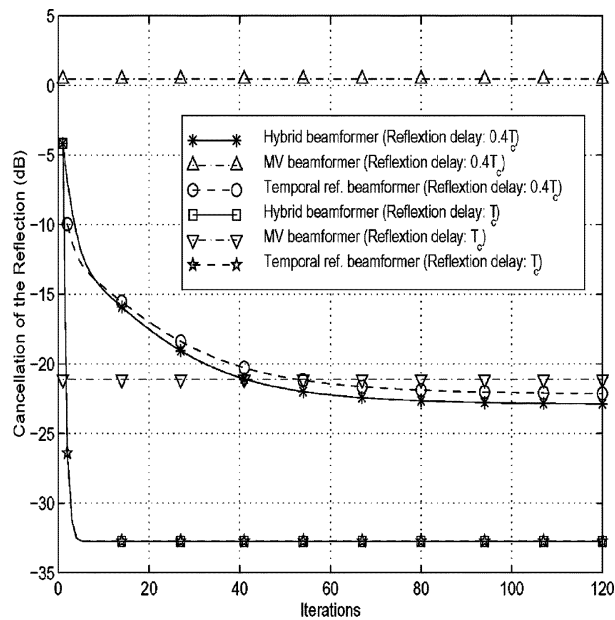

(b) Reflection cancellation

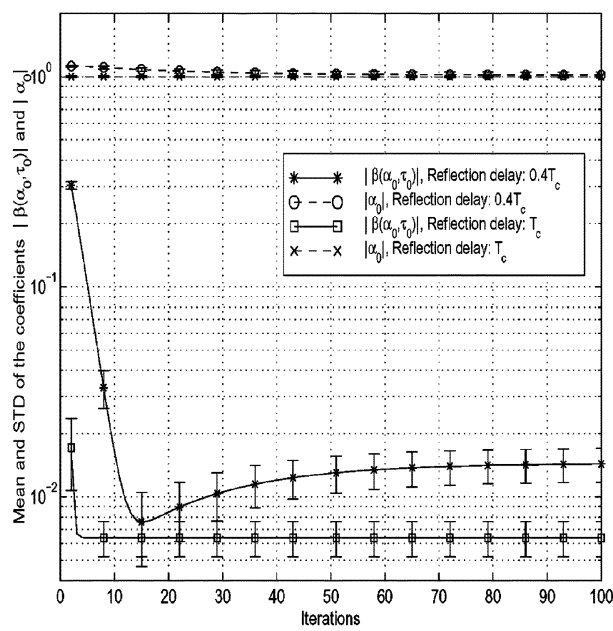

(c) Combining coefficients.

Fig. 8. Convergence of the iterative algorithm for the hybrid beamformer method in the presence of one reflection. Parameters: $\check{\theta}_{0}=0^{\circ}, \check{\tau}_{0}=0$, $\check{\theta}_{1}=10^{\circ}, \check{\tau}_{1}=\left\{0.4 T_{c}, T_{c}\right\}, \check{\alpha}_{0} / \check{\alpha}_{1}=\sqrt{2}, K=3$ pulses, $m=6$ antennas, $\mathrm{SNR}_{0}=15.87 \mathrm{~dB}$.

agree with the theoretical values predicted by (41) and (42), except for the MVBE in absence of pointing errors (this effect was already observed in Section VI-B and in [33]). The RMSE of the MVBE undergoes a severe degradation even for tiny pointing errors, whereas the MLE tolerates errors even larger than $10^{\circ}$. 


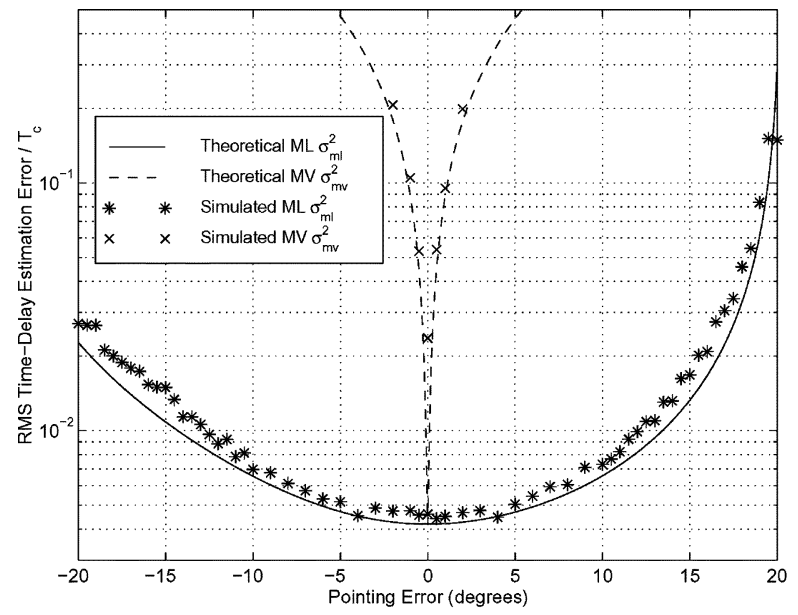

(a) One interference is received.

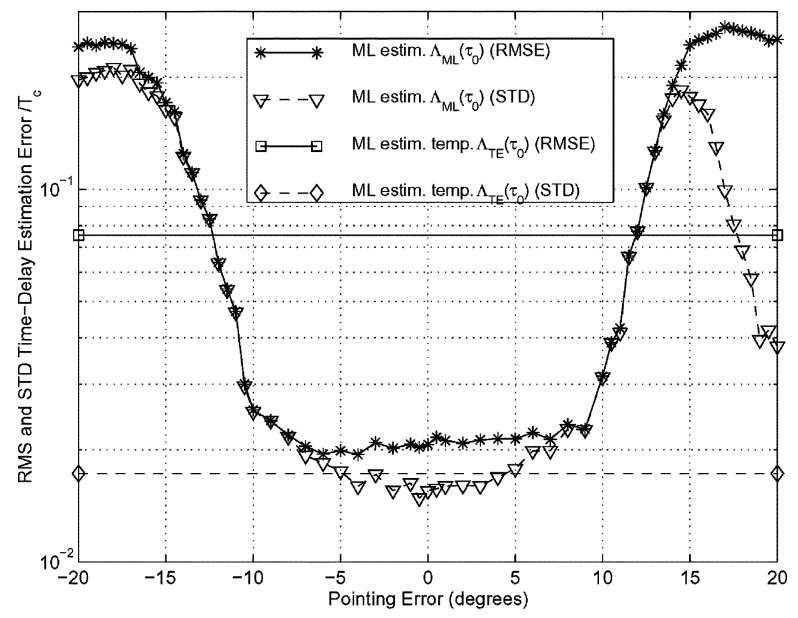

(b) One reflection is received.

Fig. 9. Effect of the pointing error on the time-delay RMSE. Parameters for (a) as in Fig. 2. Parameters for (b) as in Fig. 3 except for $\check{\tau}_{1}=0.25 T_{c}$ and $\ddot{\theta}_{1}=30^{\circ}$.

This value is related to the beamwidth of the array response, which is $19.5^{\circ}$ at the first null for the array configuration that has been employed. In Fig. 9(b), a reflection is received instead of an interference. The reflection DOA and delay are equal to $30^{\circ}$ and $0.25 T_{c}$, respectively, and the rest of parameters coincide with the default ones. The performance of the MLE is also excellent in this case; it is virtually insensitive to pointing errors up to $\pm 10^{\circ}$. Note that for pointing errors larger than $15^{\circ}$, the bias of the MLE approaches $0.25 T_{c}$. This is logical because in this case, the MLE tends to estimate the delay of the reflection as the nominal steering vector is closer to the steering vector of the reflection than to that of the direct signal.

\section{CONCLUSIONS}

An estimator of the time-delay (pseudorange) and carrierphase of the line-of-sight signal received from a GNSS satellite has been proposed. The estimator is derived by applying the ML principle to a simplified signal model, in which all components other than the desired one are modeled as a Gaussian term with unknown and arbitrary spatial correlation matrix. The estimator presented here outperforms the estimators obtained with simpler assumptions, such as that the noise is spatially white or that the spatial signature of the direct signal is unknown. Moreover, the performance of the ML estimator is very close in many situations to the best possible performance of more complex methods based on a more detailed description of the multipath channel, and it tends to be unbiased for high SNR. This is a remarkable result, taking into account that the technique has low complexity and that only the parameters of the line-of-sight signal are estimated. It also indicates that the knowledge of the LOSS steering vector makes it possible the use of the simplified model in order to derive an estimator that mitigates interferenceand multipath-induced errors and offers an excellent tradeoff between bias and RMSE for highly coherent reflections. Furthermore, this estimator is applicable in the same way in the presence of any type of multipath, which is another advantage with respect to methods based on a detailed model of the signal scenario, and it is robust against pointing or calibration errors of the antenna array. The equivalence between the ML estimator and a method based on a hybrid beamformer has been proven. This equivalence suggests possible practical implementations of the ML estimator, because it shows that the estimates can be computed by iteratively applying two different yet coupled steps: One step consists of using a spatial filter, whereas the other one is a single-antenna estimation method.

\section{REFERENCES}

[1] B. Hofmann-Wellenhof, H. Lichtenegger, and J. Collins, Global Positioning System, Theory and Practice. New York: Springer-Verlag, 1997.

[2] L. R. Weill, "Multipath mitigation using modernized GPS signals: how good can it get?," in Proc. ION-GPS, Portland, OR, Sep. 2002, pp. 493-505.

[3] R. A. Iltis and L. B. Milstein, "Performance analysis of narrow-band interference rejection techniques in DS spread-spectrum systems," IEEE Trans. Commun., vol. COM-32, no. 11, pp. 1169-1177, Nov. 1984

[4] D. Doris and A. Benhallam, "An overview of signal processing techniques to reduce multipath effects on GPS reception," in Proc. IEEE/IEE Int. Workshop Signal Process. Methods Multipath Enviroments, Glasgow, U.K., 1995.

[5] W. Zhuang and J. Tranquilla, "Effects of multipath and antenna on GPS observables," Proc. Inst. Elect. Eng.—Radar, Sonar, Navigat., vol. 142, no. 5, pp. 267-275, Oct. 1995.

[6] C. J. Comp and P. Axelrad, "Adaptive SNR-based carrier phase multipath mitigation technique," IEEE Trans. Aerosp. Electron. Syst., vol. 34, no. 1, pp. 264-276, Jan. 1998.

[7] R. D. J. van Nee, "Spread-spectrum code and carrier synchronization errors caused by multipath and interference," IEEE Trans. Aerosp. Electron. Syst., vol. 29, no. 4, pp. 1359-1365, Oct. 1993.

[8] R. D. J. van Nee, J. Siereveld, P. C. Fenton, and B. R. Townsend, "The multipath estimating delay lock loop: approaching theoretical accuracy limits," in Proc. IEEE Position, Location Navigation Symp., 1994, pp. 246-251.

[9] A. J. Van Dierendonck and M. S. Braasch, "Evaluation of GNSS receiver correlation processing techniques for multipath and noise mitigation," in Proc. ION Nat. Tech. Meet., Santa Monica, CA, Jan. 1997.

[10] L. Garin and J.-M. Rousseau, "Enhanced strobe correlator multipath rejection for code \& carrier," in Proc. ION-GPS Conf., Kansas City, MO, 1997.

[11] R. L. Fante and J. J. Vacarro, "Cancellation of Jammers and Jammer multipath in a GPS receiver," IEEE Aerosp. Electron. Syst. Mag., vol. 13, no. 11, pp. 25-28, Nov. 1998.

[12] M. D. Zoltowski and A. S. Gecan, "Advanced adaptive null steering concepts for GPS," in Proc. MILCOM Conf. Universal Commun., vol. 3, San Diego, CA, 1995, pp. 1214-1218.

[13] D. J. Moelker and Y. Bar-Ness, "An optimal array processor for GPS interference cancellation," in Proc. 15th DASC. AIAA/IEEE Digital Avionics Syst. Conf., Atlanta, GA, 1996, pp. 285-290. 
[14] G. F. Hatke, "Adaptive array processing for wideband nulling in GPS systems," in Proc. 32th Asilomar Conf. Signals, Syst., Comput., 1998, pp. 1332-1336.

[15] W. L. Myrick, M. Zoltowski, and J. S. Goldstein, "Exploting conjugate symmetry in power minimization based pre-processing for GPS: reduced complexity and smoothness," in Proc. ICASSP, Istanbul, Turkey, Jun. 2000.

[16] D. J. Moelker, "Multiple antennas for advanced GNSS multipath mitigation and multipath direction finding," in Proc. ION-GPS Meet., Kansas City, MO, 1997.

[17] G. Seco and J. A. Fernández-Rubio, "Maximum likelihood propagation-delay estimation in unknown correlated noise using antenna arrays: application to global navigation satellite systems," in Proc. ICASSP, vol. IV, Seattle, WA, May 1998, pp. 2065-2068.

[18] G. Seco, A. L. Swindlehurst, and D. Astély, "Exploting antenna arrays for synchronization," in Signal Processing Advances in Wireless Communications, G. B. Giannakis, Y. Hua, P. Stoica, and L. Tong, Eds. Englewood Cliffs, NJ: Prentice-Hall, 2000, vol. II, Trends in Single- and Multi-User Systems, ch. 10, pp. 403-430.

[19] J. K. Ray, M. E. Cannon, and P. Fenton, "Mitigation of static carrier phase multipath effects using multiple closely spaced antennas," Navigat.: J. Inst. Navigat., vol. 46, no. 3, pp. 193-201, 1999.

[20] S. Bensley and B. Aazhang, "Maximum-likelihood synchronization of a single user for code-division multiple-access communication systems," IEEE Trans. Commun., vol. 46, no. 3, pp. 392-399, Mar. 1998.

[21] A. L. Swindlehurst, "Time delay and spatial signature estimation using known asynchronous signals," IEEE Trans. Signal Process., vol. 46, no. 2, pp. 449-462, Feb. 1998.

[22] A. Jakobsson, A. L. Swindlehurst, D. Astély, and C. Tidestav, "A blind frequency domain method for DS-CDMA synchronization using antenna arrays," in Proc. 32nd Asilomar Conf. Signals, Syst., Comput., Pacific Groove, CA, Nov. 1998, pp. 1848-1852.

[23] G. W. Hein, J. Godet, J.-L. Issler, J.-C. Martin, P. Erhard, R. Lucas-Rodríguez, and T. Pratt, "Status of Galileo frequency and signal design," in Proc. ION-GPS, Portland, OR, Sep. 2002. [Online] http://europa.eu.int/comm/dgs/energy_transport/galileo/documents/technical_en.htm.

[24] M. Wax and A. Leshem, "Joint estimation of time delays and directions of arrival of multiple reflections of a known signal," IEEE Trans. Signal Process., vol. 45, no. 10, pp. 2477-2484, Oct. 1997.

[25] A.-J. van der Veen, M. C. Vanderveen, and A. Paulraj, "Joint angle and delay estimation using shift-invariance techniques," IEEE Trans. Signal Process., vol. 46, no. 2, pp. 405-418, Feb. 1998.

[26] Z.-S. Liu, J. Li, and S. L. Miller, "A receiver diversity based code-timing estimator for asynchronous DS-CDMA systems," in Proc. ICASSP, vol. VI, Seattle, WA, 1998, pp. 3245-3248.

[27] A. Ranheim and P. Pelin, "Joint symbol detection and channel parameter estimation in asynchronous DS-CDMA systems," IEEE Trans. Signal Process., vol. 48, no. 2, pp. 545-550, Feb. 2000

[28] A. L. Swindlehurst and P. Stoica, "Maximum likelihood methods in radar array signal processing," Proc. IEEE, vol. 86, no. 2, pp. 421-441, Feb. 1998.

[29] Y. Jiang, J. Li, and P. Stoica, "Array signal processing in the known waveform and steering vector case," in Proc. ICASSP, 2003, pp. V.209-V.212

[30] G. Seco, "Antenna Arrays for Multipath and Interference Mitigation in GNSS Receivers," Ph.D. dissertation, Polytech. Univ. Catalonia, Barcelona, Spain, Dec. 2000 [Online]: http://www.tdx.cesca.es/TDX0730101-124722/.

[31] E. L. Lehmann, Theory of Point Estimation. New York: Wiley, 1983.

[32] B. W. Parkinson and J. J. Spilker, Eds., Global Positioning System: Theory and Applications. Washington, DC: Amer. Inst. Aeronaut., 1996, vol. I, II, Progress in Astronautics and Aeronautics.

[33] M. Wax and Y. Anu, "Performance analysis of the minimum variance beamformer," IEEE Trans. Signal Process., vol. 44, no. 4, pp. 928-937, Apr. 1996.

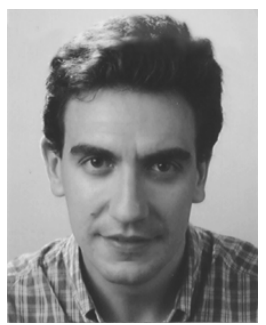

Gonzalo Seco-Granados (S'98-M'02) was born in Barcelona, Spain, in 1972. He received the M.Sc. and $\mathrm{Ph} . \mathrm{D}$. degrees in telecommunication engineering in 1996 and 2000, respectively, from Polytechnic University of Catalonia (UPC), Barcelona. He also received the MBA degree from IESE-University of Navarra, Barcelona, in 2002.

From 1997 to 2000, he was research and teaching assistant with the Signal Processing Group at UPC. Since 2002, he has been technical staff at the Electrical Engineering Department, European Space Research and Technology Center, European Space Agency, Noordwijk, The Netherlands, participating in the design of receivers for new satellite-based navigation systems. He has been with Brigham Young University, Provo, UT; Winphoria Networks, Madrid, Spain; and Columbia University, New York, NY. His research interests include signal processing for positioning and communication systems, array signal processing, synchronization, and estimation theory.

Dr. Seco-Granados received two best Ph.D. thesis awards from UPC and the Spanish Association of Telecommunication Engineers as well as the best presentation award at the ION-GPS ' 2003 conference. He was co-guest editor for a special issue of the IEEE Signal PROCESSING MAGAZINE.

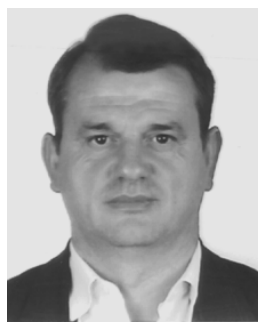

Juan A. Fernández-Rubio (S'74-M'78) was born in Macael, Spain, in 1951. He received the M.Sc.degree from the Polytechnic University of Madrid (UPM), Madrid, Spain, in 1974 and the Ph.D. degree in electrical engineering from the Polytechnic University of Catalonia (UPC), Barcelona, Spain, in 1982.

$\mathrm{He}$ has been a teacher and researcher with the UPC since 1974. He taught electromagnetic fields from 1974 until 1985, devoting his research activities to the topic of electromagnetic propagation in ferrite materials with the Department of Signal Theory and Communications, UPC, where, in 1985, he joined the Signal Processing Group, where he has been teaching signal processing and communication since 1986. $\mathrm{He}$ has also taught mathematical methods for communications, array signal processing, and communication systems for graduate students. He has also collaborated and directed some research projects funded by the European Community and the European Space Agency. He was promoted to Full Professor at UPC in 1989. His current research interests include array signal processing, wireless communications, global navigation satellite systems, audio signal processing, multiuser detection in CDMA systems, and wavelets. He has been the Director of the Technical High School of Telecommunication Engineering of Barcelona (ETSETB) since 2000, and since 2003, he has held the presidency of the Spanish Institute of Navigation (INAVE).

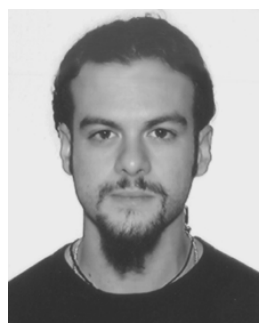

Carles Fernádez-Prades (S'02) received the M.Sc. degree in electrical engineering (with highest honors) from the Technical University of Catalonia (UPC), Barcelona, Spain, in 2000. In 2001, he was recipient of a Ministry of Education grant to work toward the Ph.D. degree at UPC.

He joined the Department of Signal Theory and Communication at UPC as a research assistant, getting involved in European (EMILY) and Spanish (TECNAV) research projects. In 2002, he was involved in an educational innovation project funded by the Catalan Government. In 2003, he became the technical manager at UPC for European Space Agency research related to the search and rescue service to be included in Galileo. His primary areas of interest include statistical and array signal processing, estimation theory, GNSS synchronization, and digital communications. 